\title{
Hierarchical Nature of the Vortex Matter in Type II Superconductors due to Competition Between Interactions, Thermal Fluctuations and Disorder
}

\author{
D. $\mathrm{Li}^{1}$ B. Rosenstein, ${ }^{2,3}$ and V. Vinokur ${ }^{4}$
}

\begin{abstract}
Published online: 9 December 2006
We describe quantitatively the combined effects of both the thermal fluctuations and of the quenched disorder via the replica trick applied to the Ginzburg-Landau (GL) theory. We show that the vortex state can appear in either of the three disordered phases: (i) unpinned vortex liquid, (ii) amorphous vortex glass (pinned), and (iii) the crystalline (pinned but not containing topological defects) Bragg glass. The formation of the vortex glass is associated with the continuous replica symmetry breaking (RSB) reflecting the hierarchial structure of the potential barriers in a vortex glass state. An earlier analysis in the framework of London approximation have established that activation barriers controlling vortex dynamics obey the extreme value statistics within roughly the same domain of the phase diagram. We show that the disordered GL model in which only the coefficient at the quadratic term $|\psi|^{2}$ is random, first considered by Dorsey et al., exhibits, in the gaussian approximation, an additional nonhierarchical state possessing certain glassy properties like nonzero Edwards-Anderson order parameter. We associate this state with the "marginal glass phase" predicted in the earlier work of one of the authors; the marginal glass state being characterized by the marginally glassy dynamics. We show further that when the random component of the coefficient of the quartic term $|\psi|^{4}$ in GL free energy is taken into account, RSB effects appear. Application of the obtained results to description of various disorder-generated phenomena in vortex matter are briefly considered. The location of the glass transition line is determined and compared to experiments. This line is clearly different from both the melting line and the second peak line describing the translational and rotational symmetry breaking at high and low temperatures respectively. The phase diagram is separated by these two lines into the four phases described above.
\end{abstract}

\section{INTRODUCTION}

Any superconductor contains inhomogeneities of either natural or artificial origin which affect both its thermodynamic and dynamic properties. The effect of inhomogeneities in the type II superconductors is described most often in terms of pinning

\footnotetext{
${ }^{1}$ School of Physics, Peking University, Beijing 100871, China.

${ }^{2}$ Electrophysics Department, National Chiao Tung University, Hsinchu 30050, Taiwan, ROC.

${ }^{3}$ Department of Condensed Matter Physics, Weizmann Institute of Science, Rehovot 76100, Israel.

${ }^{4}$ Materials Science Division, Argonne National Laboratory, Argonne, Illinois 60439, USA.
}

of the vortex lines. These effects become especially interesting in high-temperature superconductors where the interplay between disorder and thermal fluctuations gives rise to a wealth of thermodynamic and dynamic phases. While in perfectly clean superconductors, vortex system can be found in either crystalline or vortex liquid state [1], disorder can drive vortex system into a glass. Although naively one can distinguish three generic vortex phases associated with the dominance of one of the three basic energies (elastic energy, pinning energy, and the energy of thermal fluctuations), the actual vortex phase diagram reveals much more of complex diversity (see [2,3] for a review, and also [4,5]). In 
particular, there are experimental indications that there may be two distinct vortex liquid states [6]); vortex solid can form either low-field topological defects free Bragg glass phase [7] or amorphous (entangled) glassy phase [8,9]. The transition lines themselves show more complicated picture rather than simply three basic lines merging in the tri-critical point (see, for example [4]). One can summarize the existing experimental observations on the phase diagram as follows.

1. The first-order [10,11] melting line seems to merge with the so-called "second magnetization peak" line (which marks the transition from Bragg glass to amorphous glass $[8,9])[12,13]$. At low temperatures, this latter line depends strongly on disorder and generally exhibits a positive slope (termed also the "inverse" melting [14]), while in the "melting" section it is dominated by thermal fluctuations and has a large negative slope. This is the first-order transition, and the system shows entropy jump when crossing this line. The maximum at this curve where the magnetization and the entropy jumps vanish was interpreted as either a tricritical point $[6,15]$ or a Kauzmann point [16]. This universal "order-disorder" transition line (ODT), which was first observed in the strongly layered superconductors (BSCCO [12]) was extended to the moderately anisotropic superconductors ( $\mathrm{LaSCCO}$ [13]) and to the more isotropic ones like YBCO [16,17]. At this line, the spontaneous breaking of the translation and rotation symmetry occurs.

2. The universal "order-disorder" line is different from the "irreversibility line" or the "glass" transition (GT) line, which is a continuous transition $[18,19]$. The almost vertical glass line clearly represents effects of disorder, although thermal fluctuations affect the location of the transition. Experiments in $B S C C O[20]$ indicate that the line crosses the ODT line right at its maximum and continues deep into the ordered (Bragg) phase. Note that the proximity of the glass line to the Kauzmann point is not too surprising, since both of them mark the region of close competition between disorder and the thermal fluctuations effects. In more isotropic materials like $\mathrm{LaSCO}$ [21], the GT line is closer to the "melting" section of the ODT line and still crosses it. Most of the experiments [15] indicate that the GT line terminates at the "tricritical point" in the vicinity of the maximum of the ODT line. It is more difficult to characterize the nature of the GT transition as a "symmetry breaking." The common wisdom is that the "replica" symmetry is broken in the glass (either via "steps" or via "hierarchical" continuous process) as in the most of the spin glasses theories [22].

Despite the remarkable progress achieved and efforts expended [23-33] a lot of fundamental questions concerning the nature of the glass remain open and many mechanisms and subtleties of the related slow glassy dynamics are yet to be revealed, maintaining the physics of glasses as one of the major challenges in the condensed matter physics. Vortex systems offer a unique testing ground for experimental verification of theoretical concepts of glass dynamics. To establish the connection between the vortex glass and other kind of glasses (in particular, the spin glass), we explore the Ginzburg-Landau (GL) model in the presence of quenched disorder.

The application of the replica trick to the GL functional and comparison with the extreme value statistics-based phenomenological description of the vortex glass enables us to elaborate on the unifying picture of the glass dynamics and demonstrate that glassy behavior is associated with the continuous replica symmetry breaking (RSB). In the same RSB domain of the phase diagram, the relevant activation barriers obey the so-called Gumbel statistics, which is the underlying reason for the glassy dynamics (creep). This indicates the connection between the vortex glass and other glass models (especially, the models of the spin glass where the RSB is the inherent property). Applying our results to the description of vortex phase diagram, we determine the vortex glass transition line, which appears different and well separated from both the melting line and from the so-called second peak lines and present a general picture of the phase transformations in vortex matter.

The straightforward approach of introducing disorder via adding random components to all the GL coefficients gives rise to still complicated model requiring further simplifications to become treatable. Originally, the notion of vortex glass and the continuous glass transition exhibiting the conductivity glass scaling was introduced by reducing the GL description to the frustrated $X Y$ model (the gauge glass) [24,34]. In this approach, one fixes the 
amplitude of the order parameter, and disorder appears as random component of the vector potential. This model was studied by the various RG methods and has been extensively simulated numerically $[35,36]$. In analogy to the theory of spin glass, the replica symmetry is broken when crossing the GT line. The model faced several problems (see [37] for a review), in particular, for finite penetration depth $\lambda$ it does not exhibit any transition [38], also it is hard to explain sharp Bragg peaks observed in the experiments at low magnetic fields. The latter problem was bypassed by exploring the London description of the vortex state, which treats vortex system as an elastic medium consisting of interacting line-like objects subject to both the pinning potential and the thermal Langevin force $[33,39]$. The resulting models can be treated by the gaussian approximation $[7,40]$ and RG [34]. The theoretical observation then was that if the dimensionality, $D$, of the vortex system $2<D<4$, there exists a transition into a glassy phase in which the replica symmetry is broken following the "hierarchical pattern" (in $D=2$ the breaking is "one step"). Despite considerable success, the applicability of the descriptions of phase diagram based on the London approximation can be questioned within the fluctuational domain close to $T_{C}$. This concerns especially highly anisotropic layered superconductors like $B S C C O$ where the fluctuational region is wide and the very notion of the line-like vortices can be hardly applied within the vortex-liquid domain (although some of the aspects of the elastic medium approximation may still hold [41]). This calls for a more general approach based on the GL functional, which does not necessarily invoke the concept of vortex lines.

One of the most developed schemes in treating the GL model is the lowest Landau level (LLL) approximation valid close to the $H_{c 2}(T)$ line [42]. Dorsey et al. [27] studied the liquid phase adapting the dynamic approach [43], and Tesanovic and Herbut applied this scheme for study the effect of columnar defects in layered materials using supersymmetry considerations [44]. The goal of our work is to study the glass transitions in the Abrikosov state of type II superconductors using the replica treatment of the GL functional with disorder represented by the random component of the coefficients of the free energy. The LLL model including only $|\psi|^{2}$ disorder where RSB is absent within the gaussian approximation but appears at the postgaussian level was addressed in [45]. In this work, we do not go beyond the gaussian approximation, but instead con- sider the LLL model with the random component in every GL model term. The most general hierarchical homogeneous (liquid) ansatz [46] within the gaussian approximation and its stability are considered to obtain the glass transition line and to determine the nature of the transition for various values of the disorder strength of the GL coefficients. Then we place the glass line on the phase diagram of YBCO and compare our predictions with experiments and other theories.

The present paper is organized as follows. The general disordered GL model is introduced in Section 2 and the gaussian variational replica method is presented in Section 3. Next, in Section 4, we study the model with either $|\psi|^{2}$ disorder in some more detail, or the $|\psi|^{4}$ disorder in Section 5 and obtain the phase transition lines in those two cases. In Section 6, the general model containing both the $|\psi|^{2}$ disorder and the $|\psi|^{4}$ disorder is treated briefly. In the second part of the paper, we devote to discuss vortex dynamics in complimentary framework in Sections 8 and 9. In Section 10, we compare our results with the experimental data, and conclude in Section 11 by summarizing our results.

\section{DISORDER EFFECTS IN THE GINZBURG-LANDAU DESCRIPTION OF THE TYPE II SUPERCONDUCTOR}

\subsection{Ginzburg-Landau Free Energy}

We start from the Gibbs energy of the ideal homogeneous sample (no disorder):

$$
\begin{aligned}
G= & \int d x^{3} \frac{\hbar^{2}}{2 m_{\|}^{*}}\left|\partial_{z} \psi\right|^{2}+\frac{\hbar^{2}}{2 m_{\perp}^{*}}|\vec{D} \psi|^{2}+a^{\prime} \psi^{*} \psi \\
& +\frac{b^{\prime}}{2}\left(\psi^{*} \psi\right)^{2}+\frac{(H-B)^{2}}{8 \pi} .
\end{aligned}
$$

Here $a^{\prime}=\alpha\left(T-T_{\mathrm{c}}\right)$ and $b^{\prime}$ are constant parameters, $\vec{D} \equiv\left(-i \hbar \nabla+\frac{e^{*}}{c} \vec{A}\right)$ is the covariant derivative, $\vec{A}$ is the vector potential, the magnetic field $\vec{A}=\nabla \times \vec{A}, H$ is the external magnetic field, $m_{\perp}^{*}$ and $m_{\|}^{*}$ are the effective masses in directions perpendicular and parallel to the field respectively. Mesoscopic thermal fluctuations are accounted for via Boltzmann weights

$$
Z=\int_{\psi^{*}, \psi} \exp \left\{-\frac{G\left[\psi^{*}, \psi\right]}{T}\right\}
$$

The model provides a good description of thermal fluctuations as long as $1-t-b \ll 1$, where 
$t=T / T_{\mathrm{c}}, \quad b=B / H_{c 2}\left(h=H / H_{c 2}\right), \quad H_{c 2}=\Phi_{0} /\left(2 \pi \xi^{2}\right)$ and $\xi$ is the coherence length. In this case, the higher order terms like $|\psi|^{6}$ can be omitted (detail notation can be found in [47]). The threedimensional GL model describes materials with not too high anisotropy (for a recent evidence of validity of this assumption in $Y B C O$ see [48]). In strongly anisotropic materials, a model of the LawrenceDoniach type is more appropriate [2].

Within the GL approach, the point-like quenched disorder on the mesoscopic scale is introduced by making each of the coefficients a random variable centered around a certain constant value given in Eq. (2) with the final dispersion. For example, the effective masses assume the form

$$
\begin{aligned}
& \frac{m_{\perp}^{*-1}}{U(x) U(y)} \rightarrow m_{\perp}^{*-1}(1+U(x)) ; \\
& P \delta(x-y) .
\end{aligned}
$$

The parallel effective mass $m_{\|}^{*}$ might also have the random component which we neglect (it is relatively small, since $m_{\|}^{*}$ is typically very large), though it can be incorporated with no additional difficulties. This type of disorder is sometimes called the $\delta l$ disorder, since it originates in part from the inhomogeneity of the electron mean free path $l$ in Gor'kov's derivation. From the BCS theory, effective mass is

$$
m^{*}=2 m_{e}\left(1+\frac{\pi^{3} h v_{F}}{168 \zeta(3) T_{\mathrm{c}} l}\right)
$$

in the clean limit and

$$
m^{*}=2 m_{e} \frac{7 \zeta(3) \hbar v_{F}}{2 \pi^{3} T_{\mathrm{c}} l}
$$

in the dirty limit. Relation to the notations of [2, chapter II] is as follows: $U$ is $-\delta m_{a b} / m_{a b}$ and $P=\gamma_{m} / m_{a b}^{2}$. Note, however, that in addition to the random distribution of $l$, disorder in $v_{\mathrm{F}}$ and $T_{\mathrm{c}}$ (the density of states and interaction strength) can also affect $m^{*}$.

The other two parameters in the GL equations are

$$
\alpha=\frac{12 \pi^{2} T_{\mathrm{c}}}{7 \zeta(3) \epsilon_{F}}
$$

and

$$
b^{\prime}=\frac{18 \pi^{2}}{7 \zeta(3) N \epsilon_{F}}\left(\frac{T_{\mathrm{c}}}{\epsilon_{F}}\right)^{2} .
$$

The coefficient of the quadratic term is called $\delta T$ disorder, since it describes a local deviation of the critical temperature. Introducing a random component in $|\psi|^{2}$ term:

$$
a^{\prime} \rightarrow a^{\prime}(1+W(x)) ; \quad \overline{W(x) W(y)}=R \delta(x-y) .
$$

In notations of [2], the random field $W(x)=$ $-\delta_{a} / a, R=\gamma_{a} / a^{2}$. When thermal fluctuations of the vortex degrees of freedom can be neglected, these two random fields would be sufficient (they control the two relevant scales $\xi$ and $\lambda$ ). The reason is that one can set the coefficient of the third term $|\psi|^{4}$ to a constant by rescaling. However, in the presence of thermal fluctuations, the coefficient of $|\psi|^{4}$ also should be considered as having a random component. It cannot be "rescaled out," since it affects the Boltzmann weights. Later we will see that at least within the LLL approximation, this term is crucial in inducing certain glassy properties of the vortex matter state. We, therefore, introduce its disorder via

$$
b^{\prime} \rightarrow b^{\prime}(1+V(x)) ; \quad \overline{V(x) V(y)}=Q \delta(x-y) .
$$

In unconventional superconductors, even without disorder, the phenomenological GL model has not been reliably derived microscopically. The coefficients and their inhomogeneitics therefore should be considered as phenomenological parameters to be fitted to experiments. We assume that $U, R$, and $Q$ have weak dependencies on field and temperature. The assumption of the weak temperature and field dependence of the disorder strengths $U, R$, and $Q$, as that of any parameter in the GL approach, should be derived in principal from a microscopic theory assuming random chemical potential or should be justified by fitting to experiments. For simplicity, the white noise distribution is considered

$$
p[U, W, V]=\exp \left[-\int_{x} \frac{U(x)^{2}}{2 P}+\frac{W(x)^{2}}{2 R}+\frac{V(x)^{2}}{2 Q}\right]
$$

for random components. The free energy of superconductor after averaging over the disorder is

$$
\begin{aligned}
\bar{F}= & -\frac{T}{\operatorname{norm}} \int_{U, W, V} p[U, W, V] \log \left[\int_{\psi} \exp [-g[\psi]\right. \\
& \left.\left.-f_{\mathrm{dis}}[U, W, V, \psi]\right]\right] ; \\
g= & G / T ; f_{\mathrm{dis}}[U, W, V, \psi]=\frac{1}{T} \int_{x} \frac{\hbar^{2}}{2 m_{\perp}^{*}} U(x)|\vec{D} \psi|^{2} \\
& +a^{\prime} W(x)|\psi|^{2}+\frac{1}{2} V(x)|\psi|^{4},
\end{aligned}
$$

where

$$
\text { norm } \equiv \int_{U, W, V} p[U, W, V]
$$

is a normalization factor. 
To make the physical picture clear, we rescale the coordinates as $x \rightarrow \xi x, y \rightarrow \xi y, z \rightarrow \frac{\xi z}{\gamma}$ with anisotropy parameter defined by $\gamma=\left(m_{\|}^{*} / m_{\perp}^{*}\right)^{1 / 2}$. The order parameter is scaled as $\psi^{2} \rightarrow \frac{2 \alpha T_{\mathrm{c}}}{b^{\prime}} \psi^{2}$. The dimensionless free energy becomes simpler looking:

$$
\begin{aligned}
g[\psi]= & G / T=\frac{1}{\omega} \int_{x} \frac{1}{2}\left|\partial_{z} \psi\right|^{2}+\frac{1}{2}|\vec{D} \psi|^{2}+\frac{t-1}{2}|\psi|^{2} \\
& +\frac{1}{2}|\psi|^{4}+\frac{\kappa^{2}(b-h)^{2}}{4},
\end{aligned}
$$

where $\omega=\sqrt{2 G i} \pi^{2} t$. The Ginzburg number is $G i \equiv$ $32\left[\pi \lambda^{2} T_{\mathrm{c}} \gamma /\left(\Phi_{0}^{2} \xi\right)\right]^{2}$, where $\lambda$ is the magnetic penetration depth. The last term can be ignored in calculating $\bar{F}$ as the $\kappa$ is very big in high $T_{\mathrm{c}}$ superconductors and the last term is order of $\frac{1}{\kappa^{2}}$. Similarly, the random component and the distribution become:

$$
\begin{aligned}
f_{\mathrm{dis}}[U, W, V, \psi]= & \frac{1}{\omega} \int_{x}\left\{-\frac{1}{2} U(x) \psi^{*} D^{2} \psi+\frac{t-1}{2} W(x)|\psi|^{2}\right. \\
& \left.+\frac{1}{2} V(x)|\psi|^{4}\right\} . \\
p[U, W, V]= & \exp \left[-\frac{\xi^{3}}{\gamma} \int_{x}\left(\frac{U(x)^{2}}{2 P}+\frac{W(x)^{2}}{2 R}\right.\right. \\
& \left.\left.+\frac{V(x)^{2}}{2 Q}\right)\right] .
\end{aligned}
$$

The model, however, is highly nontrivial even without disorder, and further approximation is needed to make progress.

\subsection{Lowest Landau Level Approximation}

The LLL approximation [42] is based on constraint $-D^{2} \psi=b \psi$ Over the years, this model has been studied by various methods, analytic and numerical $[47,49,50]$. The (effective) LLL model is applicable in a surprisingly wide range of fields and temperatures determined by the condition that the relevant excitation energy $\varepsilon$ is much smaller than the gap between Landau levels $2 \hbar e B /\left(\mathrm{cm}_{\perp}\right)$ [16].

The free energy after further rescaling $x \rightarrow$ $x / \sqrt{b}, y \rightarrow y / \sqrt{b}, z \rightarrow z\left(2^{5 / 2} \pi / b \omega\right)^{1 / 3}, \psi^{2} \rightarrow\left(2^{5 / 2} \pi /\right.$ $\left.b_{\omega}\right)^{2 / 3} \psi^{2}$, simplifies within the LLL approximation to:

$$
f_{\mathrm{LLL}}=\frac{1}{2^{5 / 2} \pi} \int d^{3} x\left[\frac{1}{2}\left|\partial_{z} \psi\right|^{2}+a_{T}|\psi|^{2}+\frac{1}{2}|\psi|^{4}\right] .
$$

Not surprisingly, the number of independent constants in LLL is one less than in the general model.
This fact leads to the "LLL scaling" relations (of course, the disorder terms will break LLL scaling). As a result, the simplified model without disorder has just one parameter-the (dimensionless) scaled temperature:

$$
a_{T}=-\left(\frac{2 \pi}{b \omega}\right)^{2 / 3}(1-t-b) .
$$

The disorder term becomes:

$$
f_{\mathrm{LLL}}^{\mathrm{dis}}=\frac{1}{2^{5 / 2} \pi} \int d^{3} x\left\{\Omega(x)|\psi|^{2}+\frac{1}{2} V(x)|\psi|^{4}\right\},
$$

in which only combination of $W$ and $U$ enters

$$
\begin{aligned}
\Omega(x)= & \frac{1}{2}\left[2(t-1)\left(\frac{2 \pi}{b \omega}\right)^{2 / 3} W(x)\right. \\
& \left.-2 b\left(\frac{2 \pi}{b \omega}\right)^{2 / 3} U(x)\right] .
\end{aligned}
$$

Its distribution is still gaussian

$$
\bar{p}(\Omega, V)=\exp \left[-\int_{x} \frac{\Omega(x)^{2}}{2 r^{\prime}}+\frac{V(x)^{2}}{2 q}\right]
$$

with two variances

$$
\begin{aligned}
& r^{\prime}=\frac{\sqrt{2} \gamma \pi}{\omega \xi^{3}}\left\{(1-t)^{2} R+b^{2} P\right\} \\
& q^{\prime}=\frac{\gamma}{\sqrt{2} \xi^{3}}\left(\frac{b^{2} \omega}{2 \pi}\right)^{1 / 3} Q .
\end{aligned}
$$

To treat both the thermal fluctuations and disorder, we will use the replica method to integrate over impurity distribution followed by gaussian approximation.

\section{REPLICA TRICK AND GAUSSIAN APPROXIMATION}

\subsection{Replica Trick}

We will use the replica trick to evaluate the disorder averages. The replica method is widely used to study disordered electrons in the theory of spin glasses [22], disordered metals and was applied to vortex matter in the London limit [40,51]. Applying a simple mathematical identity to the disorder average of the free energy, one obtains:

$$
\bar{F}=\overline{-T \lim _{n \rightarrow 0} \frac{1}{n}\left(Z^{n}-1\right)} .
$$


The averages of $Z^{n}$ is the statistical sum over $n$ identical "replica" fields $\psi_{a}, a=1, \ldots, n$ :

$$
\begin{aligned}
\overline{Z^{n}}= & \frac{1}{\operatorname{norm}} \int_{\Omega, V} p[\Omega, V] \prod_{a} \int_{\psi_{a}} \exp \left\{-f\left[\psi_{a}\right]\right. \\
& \left.-f_{\text {dis }}\left[\Omega, V, \psi_{a}\right]\right\} .
\end{aligned}
$$

The integral over the disorder potential is gaussian and results in:

$$
\begin{aligned}
& \overline{Z^{n}}=\int_{\psi_{a}} \exp \left[-\sum_{a} f\left(\psi_{a}\right)+\frac{1}{2\left(2^{5 / 2} \pi\right)^{2}} \sum_{a, b} f_{a b}\right] \\
& f_{a b}=r^{\prime}\left|\psi_{a}\right|^{2}\left|\psi_{b}\right|^{2}+\frac{q^{\prime}}{4}\left(\psi_{a}^{*} \psi_{a}\right)^{2}\left(\psi_{b}^{*} \psi_{b}\right)^{2} .
\end{aligned}
$$

This model is a type of scalar field theory and the simplest nonperturbative scheme commonly used to treat such a model is gaussian approximation. Its validity and precision can be checked only by calculating corrections.

\subsection{Gaussian Approximation}

We have assumed that the order parameter is constrained to the LLL and therefore can be expanded on the basis of the standard LLL eigenfunctions in Landau gauge:

$$
\psi_{a}(x)=\operatorname{norm} \int_{k_{z}, k} e^{i\left(z k_{z}+x k\right)} \exp \left\{-\frac{1}{2}(y+k)^{2}\right\} \tilde{\psi}_{a}(k) .
$$

We now apply the gaussian approximation, which has been used in disorder in the elastic medium approach [40,51], following its use in polymer physics [52]. The gaussian approximation was applied to the vortex liquid within the GL approach in $[42,50]$. The gaussian effective free energy is expressed via variational parameter [47,52] $\mu_{a b}$, which, in the present case, is a matrix in the replica space. The correlator is parameterized as follows

$$
\left\langle\psi_{a}^{*}\left(k, k_{z}\right) \psi_{b}\left(-k,-k_{z}\right)\right\rangle=G_{a b}\left(K_{z}\right)=\frac{2^{5 / 2} \pi}{\frac{k_{z}^{2}}{2} \delta_{a b}+\mu_{a b}^{2}}
$$

The bubble integral appearing in the free energy is very simple:

$$
\begin{aligned}
\left\langle\psi_{a}^{*}(x, y, z) \psi_{b}(x, y, z)\right\rangle & =\frac{\sqrt{2}}{\pi} \int_{k_{z}} \frac{1}{\frac{k_{z}^{2}}{2} \delta_{a b}+\mu_{a b}^{2}} \\
& =2 \mu_{a b}^{-1} \equiv 2 m_{a b} .
\end{aligned}
$$

As a result, the gaussian effective free energy can be written in a form:

$$
\begin{aligned}
n f_{\text {eff }}= & \sum_{a}\left\{\frac { 2 ^ { 5 / 2 } \pi } { ( 2 \pi ) ^ { 3 } } \int _ { k _ { z } } \left[\log G^{-1}\left(k_{z}\right)\right.\right. \\
& \left.\left.+\left(\frac{k_{z}^{2}}{2}+a r\right) G\left(k_{z}\right)-I\right]_{a a}+4\left(m_{a a}\right)^{2}\right\} \\
& -\sum_{a, b}\left\{\frac{1}{2^{3 / 2} \pi} r^{\prime}\left|m_{a b}\right|^{2}+\frac{\sqrt{2}}{\pi} q^{\prime}\left(\left|m_{a b}\right|^{4}\right.\right. \\
& \left.\left.+4 m_{a a} m_{b b}\left|m_{a b}\right|^{2}\right)\right\} \\
= & 2 \sum_{a}\left\{\mu_{a a}+a_{T} m_{a a}+2\left(m_{a a}\right)^{2}\right\} \\
& -2 \sum_{a, b}\left\{r\left|m_{a b}\right|^{2}+q\left(\frac{1}{4}\left|m_{a b}\right|^{4}\right.\right. \\
& \left.\left.+m_{a a} m_{b b}\left|m_{a b}\right|^{2}\right)\right\},
\end{aligned}
$$

where we discarded an (ultraviolet divergent) constant and renormalization of $a_{T}$ and rescaled the disorder strength: $r=\left[1 /\left(2^{5 / 2} \pi\right)\right] r^{\prime}, q=2^{5 / 2} / \pi q^{\prime}$.

We start with a simple case in which only the $|\psi|^{2}$ type of disorder is present. More precisely, we take $q=0$ and return to the general case in Section 4. This model has been already discussed using different method (the Sompolinsky dynamic approach) in the unpinned phase in [27].

\section{NONZERO EDWARDS-ANDERSON ORDER PARAMETER AND ABSENCE OF THE REPLICA SYMMETRY BREAKING WHEN ONLY THE $|\psi|^{2}$ DISORDER IS PRESENT}

\subsection{Hierarchical Matrices and Impossibility of the Continuous Replica Symmetry Breaking}

In this section, we neglect the $|\psi|^{4}$ disorder term. It is convenient to introduce real (not necessarily symmetric) matrix $Q_{a b}$, which is in one to one linear correspondence with Hermitian (generally complex) matrix $m_{a b}$ via

$$
Q_{a b}=\operatorname{re}\left[m_{a b}\right]+\operatorname{im}\left[m_{a b}\right] .
$$

Unlike $m_{a b}$, all the matrix elements of $Q_{a b}$, are independent. In terms of this matrix, the free energy can 
be written as

$$
\frac{n}{2} f_{\mathrm{eff}}=\sum_{a}\left\{\left(m^{-1}\right)_{a a}+a_{T} Q_{a a}+2\left(Q_{a a}\right)^{2}\right\}-r \sum_{a, b} Q_{a b}^{2} .
$$

Taking derivative with respect to $Q_{a b}$, gives the saddle point equation for this matrix element:

$$
\begin{aligned}
\frac{n}{2} \frac{\delta f}{\delta Q_{a b}}= & -\frac{1}{2}\left[(1-i)\left(m^{-2}\right)_{a b}+\text { c.c. }\right]+a_{T} \delta_{a b} \\
& +4 Q_{a a} \delta_{a b}-2 r Q_{a b}=0 .
\end{aligned}
$$

Since the electric charge (or the superconducting phase) $U(1)$ symmetry is assumed, we consider only solutions with real $m_{a b}$. In this case, $m_{a b}=Q_{a b}$ is a symmetric real matrix. General hierarchical matrices $m$ are parameterized using the diagonal elements $\tilde{m}$ and the Parisi's (monotonically increasing) function $m_{x}$ specifying the off diagonal elements with $0<x<1$ [52]. Physically different $x$ represent time scales in the glass phase. In particular, the EdwardsAnderson (EA) order parameter is $m_{x=1}=M>0$.

A nonzero value for this order parameter signals that the annealed and the quenched averages are different. The dynamic properties of such phase are generally quite different from those of the nonglassy $M=0$ phase. In particular, it is expected to exhibit infinite conductivity [24,27]. We will refer to this phase as the "ergodic pinned liquid" (EPL) distinguished from the "nonergodic pinned liquid" (NPL) in which, in addition, the ergodicity is broken.

However, in the present model, RSB does not occur. In terms of Parisi parameter $\tilde{m}$ and $m_{x}$, the matrix equation (24) takes a form:

$$
\begin{aligned}
-\widetilde{m^{-2}}+a_{T}+(4-2 r) \tilde{m} & =0 \\
\left(m^{-2}\right)_{x}+2 r m_{x} & =0 .
\end{aligned}
$$

Dynamically, if $m_{x}$ is a constant, pinning does not results in the multitude of time scales. Certain time scale sensitive phenomena like various memory effects [53] and the responses to "shaking" [14] are expected to be different from the case when $m_{x}$ takes multiple values. If $m_{x}$ takes a finite different number of $n$ values, we call $n-1$ step RSB. However, if $m_{x}$ is continuous, the continuous RSB occurs.

In order to show that $m_{x}$ is a constant, it is convenient to rewrite the second equation via the matrix $\mu$, the matrix inverse to $m$ :

$$
\left(\mu^{2}\right)_{x}+2 r\left(\mu^{-1}\right)_{x}=0
$$

Differentiating this equation with respect to $x$, one obtains:

$$
2\left[\{\mu\}_{x}-r\left(\{\mu\}_{x}\right)^{-2}\right] x \frac{d \mu_{x}}{d x}=0,
$$

where we used a set of standard notations in the spin glass theory [52]:

$$
\begin{gathered}
\{\mu\}_{x} \equiv \tilde{\mu}-\left\langle\mu_{x}\right\rangle-[\mu]_{x} ; \quad\left\langle\mu_{x}\right\rangle \equiv \int_{0}^{1} d x \mu_{x} ; \\
{[\mu]_{x}=\int_{0}^{x} d y\left(\mu_{x}-\mu_{y}\right) .}
\end{gathered}
$$

If one is interested in a continuous monotonic part $d \mu_{x} / d x \neq 0$, the only solution of Eq. (27) is

$$
\{\mu\}_{x}=r^{1 / 3}
$$

Differentiating this again and dropping the nonzero derivative $d \mu_{x} / d x$ again, one further gets a contradiction: $d \mu_{x} / d x=0$. This proves that there are no such monotonically increasing continuous segments. One can therefore generally have either the replica symmetric solutions, namely $m_{x}=M$ or look for a several step-like RSB solutions [22]. We can show that the constant $m_{x}$ solution is stable. Therefore, if a steplike RSB solution exists, it might be only an additional local minimum. We explicitly looked for a onestep solution and found that there is none.

\subsection{Two Replica symmetric solutions and the Third-Order Transition Between Them}

\subsubsection{The Unpinned Liquid and the "Ergodic Glass" Replica Symmetric Solutions}

Restricting to RS solutions, $m_{x}=M$, the saddle point equations (25) simplify:

$$
\begin{aligned}
-\varepsilon^{-2}+\left(a_{T}+4 \widetilde{m}\right)-2 r \varepsilon & =0 ; \\
M\left(\varepsilon^{-3}-r\right) & =0,
\end{aligned}
$$

where $\epsilon=\widetilde{m}-M$. Energy of such a solution is given by

$$
\begin{aligned}
\frac{f_{\text {eff }}}{2}= & 2 \varepsilon^{-1}-2-\varepsilon^{-2} M+2 a_{T} \tilde{m}+4 \widetilde{m}^{2} \\
& -2 r\left(\varepsilon^{2}+2 \varepsilon M\right) .
\end{aligned}
$$

The second equation (30) has a replica index independent (diagonal) solution $M=0$. In addition, there is a nondiagonal one. It turns out that there is a third-order transition between them. 
For the diagonal solution $\varepsilon=\widetilde{m}$, and the first equation is just a cubic equation:

$$
-\widetilde{m}^{-2}+\left(a_{T}+4 \widetilde{m}\right)-2 r \tilde{m}=0 .
$$

For the nondiagonal solution, the second equation gives $\varepsilon=r^{1 / 3}$, which, when plugged into the first equation, gives:

$$
\widetilde{m}=\frac{1}{4}\left(3 r^{2 / 3}-a_{T}\right) ; \quad M=\frac{1}{4}\left(3 r^{2 / 3}-a_{T}\right)-r^{-1 / 3} .
$$

The matrix $m$ therefore is

$$
m_{a b}=r^{-1 / 3} \delta_{a b}+M,
$$

which results in the following value of the free energy:

$$
f=6 r^{1 / 3}-\frac{1}{4}\left(3 r^{2 / 3}-a_{T}\right)-r^{2} .
$$

The two solutions coincide for

$$
a_{T}=r^{-1 / 3}(3 r-4) .
$$

Since in addition to the energy, the first and second derivatives of the energy, $\left(d f / d a_{T}\right)=\left(2 r^{-1 / 3}\right)$ and $\left(d^{2} f / d a_{T}^{2}\right)=-(1 / 2)$, respectively, coincide (the fourth derivatives are different though), the transition is a third-order one.

\subsubsection{Stability Domains of the Two Solutions}

In order to prove that a solution is stable beyond the set of replica symmetric matrices $m$, one has to calculate the second derivative of free energy (called Hessian in [22,54]) with respect to arbitrary real matrix $Q_{a b}$ defined in Eq. (22):

$$
\begin{aligned}
H_{(a b)(c d) \equiv} \equiv & \frac{n}{2} \frac{\delta^{2} f_{\text {eff }}}{\delta Q_{a b} \delta Q_{c d}} \\
= & \frac{1}{2}\left[\left(m^{-2}\right)_{a c}\left(m_{d b}^{-1}\right)-i\left(m^{-2}\right)_{a d}\left(m^{-1}\right)_{c b}\right] \\
& +\frac{1}{2}\left[\left(m^{-1}\right)_{a c}\left(m^{-2}\right)_{d b}-i\left(m^{-1}\right)_{a d}\left(m^{-2}\right)_{c b}\right]+\text { c.c. } \\
& +4 \delta_{a c} \delta_{b d} \delta_{a b}-2 r \delta_{a c} \delta_{b d} .
\end{aligned}
$$

We will use a simplified notation for the product of the Kronecker delta functions with more than two indices: $\delta_{a c} \delta_{b d} \delta_{a b} \equiv \delta_{a b c d}$. For the diagonal solution, the Hessian is a very simple operator on the space of real symmetric matrices:

$$
H_{(a b)(c d)}=c_{I} I_{a b c d}+c_{J} J_{a b c d},
$$

where the operators $I$ (the identity in this space) and $J$ are defined as

$$
I \equiv \delta_{a c} \delta_{b d} ; \quad J=\delta_{a b c d}
$$

and their coefficients in the diagonal phase are:

$$
c_{I}=2\left(\widetilde{m}^{-3}-r\right), \quad c_{J}=4
$$

with $\widetilde{m}$ being a solution of Eq. (32). The corresponding eigenvectors in the space of symmetric matrices are $v_{(c d)} \equiv A \delta_{c d}+B$. To find eigenvalues $\lambda$ of $H$, we apply the Hessian on $V$. The result is (dropping terms vanishing in the limit $n \rightarrow 0$ ):

$$
\begin{aligned}
H_{(a b)(c d) v_{c d}} & =A\left(c_{I}+c_{J}\right) \delta_{a b}+B\left(c_{I}+c_{J} \delta_{a b}\right) \\
& =\lambda\left(A \delta_{a b}+B\right)
\end{aligned}
$$

There are two eigenvalues: $\lambda^{(1)}=c_{I}$ and $\lambda^{(2)}=c_{I}+$ $c_{J}$. Since $c_{J}=4>0$, the sufficient condition for stability is:

$$
c_{I}=2\left(\tilde{m}^{-3}-r\right)>0 .
$$

It is satisfied everywhere below the transition line of Eq. (35), see Fig. 1 ( $a_{T}, r$ phase diagram). The analysis of stability of the nondiagonal solution is slightly more complicated. The Hessian for the nondiagonal solution is:

$$
H_{(a b)(c d)}=c_{V} V+c_{U} U+c_{j} J
$$

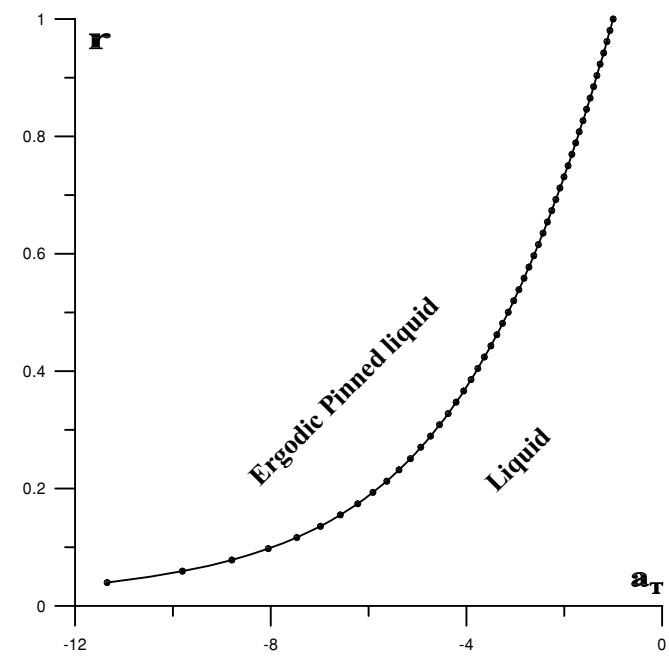

Fig. 1. Schematic $a_{T}-r$ plane phase diagram of the vortex liquid with the $|\psi|^{2}$ disorder only. $a_{T}$ is the LLL scaled temperature, while $r$ is the $|\psi|^{2}$ disorder strength. The dotted line is the glass transition line. Below the line, the state is described by a replica diagonal matrix $m_{a b}=m \delta_{a b}$, while above line, the vortex state has a nonzero Anderson Edwards parameter. 
where new operators are

$$
V_{(a b)(c d)}=\delta_{a c}+\delta_{b d} ; \quad U_{(a b)(c d)}=1
$$

and coefficients are

$$
c_{V}=-3 M r^{2 / 3} ; \quad c_{U}=4 M^{2} r^{1 / 3} ; \quad c_{J}=4
$$

In the present case, one obtains three different eigenvalues $[22,54], \lambda^{(1.2)}=2\left(1 \pm \sqrt{1-4 M r^{2 / 3}}\right)$ and $\lambda^{(3)}=0$. Note that the eigenvalue of Hessian on the antisymmetric matrices are degenerate with eigenvalue $\lambda^{(1)}$ in this case (we will come back later on this eigenvalue). For $M<0$, the solution is unstable due to negative $\lambda^{(2)}$. For $M>0$, both eigenvalues are positive and the solution is stable. The line $M=0$ coincides with the third-order transition line, hence the nondiagonal solution is stable when the diagonal is unstable and vise versa. Figure 1 shows phase transition between two liquids. We conclude that there is no glass state in the vortex liquid without the $|\psi|^{4}$ disorder term. The transition does not correspond to RSB. Despite this in the phase with nonzero EA (NEA), M order parameter there are Goldstone bosons corresponding to $\lambda^{(3)}$ in the replica limit of $n \rightarrow 0$. The criticality and the zero modes due to disorder (pinning) in this phase might lead to great variety of interesting phenomena in statics and dynamics. These have not been explored yet. However, as we show in the next section, the random component of the quartic term changes the character of the transition line: the replica symmetry is broken on the one side of the line. For simplicity, in the next section, we consider first a case with a random component of $|\psi|^{4}$ and no random component of $|\psi|^{2}$, and return to the general case in Section 5.

\section{THE GLASS TRANSITION FOR THE $|\psi|^{4}$ DISORDER}

\subsection{Continuous Replica Symmetry Breaking Solutions}

In this section, we neglect the $r|\psi|^{2}$ term disorder. Although it is always present, as we have seen in the previous section, at least within the gaussian approximation, it does not cause RSB. Therefore, one expects that although it certainly influences properties of the vortex matter, for example, the melting transition line to lower fields and temperatures [16], its role in qualitative understanding of RSB effects is minor. The only other disordered term within the LLL approximation considered in this paper is the $|\psi|^{4}$ disorder term. As was discussed in Section 2, at least within the BCS theory, it is expected to be smaller than the $|\psi|^{2}$ disorder, $q \ll r$. Even it could be very small, however, as we show here, it leads to qualitatively new phenomena in vortex matter. The $r=0$ free energy after integration over $k_{z}$ becomes:

$$
\begin{aligned}
\frac{n}{2} f_{\text {eff }}= & \sum_{a}\left\{\left(m^{-1}\right)_{a a}+a_{T} m_{a a}+2\left(m_{a a}\right)^{2}\right\} \\
& -q \sum_{a, b}\left(\frac{1}{4}\left|m_{a b}\right|^{4}+m_{a a} m_{b b}\left|m_{a b}\right|^{2}\right)
\end{aligned}
$$

In terms of the real matrix $Q_{a b}$ defined in Eq. (22), the free energy can be written as

$$
\begin{aligned}
& \frac{n}{2} f_{\text {eff }}=\sum_{a}\left\{\left(m^{-1}\right)_{a a}+a_{T} Q_{a a}+2\left(Q_{a a}\right)^{2}\right\} \\
& -q \sum_{a, b}\left(\frac{1}{8} Q_{a b}^{4}+\frac{1}{8} Q_{a b}^{2} Q_{b a}^{2}+Q_{a a} Q_{b b} Q_{a b}^{2}\right)
\end{aligned}
$$

Taking a derivative with respect to $Q_{a b}$ gives the saddle point equation for this matrix:

$$
\frac{n}{2} \frac{\delta f}{\delta Q_{a b}}=\left[\begin{array}{l}
-\frac{1}{2}\left[(1-i)\left(m^{-2}\right)_{a b}+c c\right]+a_{T} \delta_{a b} \\
+4 Q_{a a} \delta_{a b} \\
-q\left(\frac{1}{2} Q_{a b}^{3}+\frac{1}{2} Q_{a b} Q_{b a}^{2}+2 Q_{a b} Q_{a a} Q_{b b}\right. \\
\left.+\delta_{a b} \Sigma_{e} Q_{e c}\left(Q_{a e}^{2}+Q_{e a}^{2}\right)\right)
\end{array}\right]=0(48)
$$

Using the hierarchical symmetric matrix parametrization of its symmetric part (the antisymmetric will not be important for most of our purposes), it takes a form

$$
\begin{aligned}
-\widetilde{m^{-2}}+a_{T}+4 \tilde{m}-q\left(3 \tilde{m}^{3}+2 \widetilde{m^{2}} \widetilde{m}\right) & =0 \\
\left(m^{-2}\right)_{x}+q\left(m_{x}^{3}+2 \widetilde{m}^{2} m_{x}\right) & =0
\end{aligned}
$$

As in the previous section, it is convenient to rewrite the second equation in terms of $\mu$, the inverse matrix of $m$ :

$$
\left(\mu^{2}\right)_{x}+q\left[\left(\left(\mu^{-1}\right)_{x}\right)^{3}+2 \tilde{m}^{2}\left(\mu^{-1}\right)_{x}\right]=0 .
$$

Differentiation of this equation with respect to $x$, leads to:

$$
\left\{2\{\mu\}_{x}-q\left[3\left(\left(\mu^{-1}\right)_{x}\right)^{2}+2 \tilde{m}^{2}\right]\left(\{\mu\}_{x}\right)^{-2}\right\} x \frac{d \mu_{x}}{d x}=0 .
$$

For a continuous segment $\left(d \mu_{x} / d x\right) \neq 0$ one solves Eq. (52) for $\left(\mu^{-1}\right)_{x}$ in terms of $\{\mu\}_{x}$ getting now a more complicated result:

$$
\left(\mu^{-1}\right)_{x}=\sqrt{\frac{2}{3}\left[q^{-1}\left(\{\mu\}_{x}\right)^{3}-\tilde{m}^{2}\right]}
$$


Differentiating this equation with respect to $x$ again, one obtains:

$$
\frac{1}{\left(\{\mu\}_{x}\right)^{2}}=\sqrt{\frac{2}{3 q\left[\left(\{\mu\}_{x}\right)^{3}-q \tilde{m}^{2}\right]}}\left(\{\mu\}_{x}\right)^{2} x .
$$

Instead of solving this for $\{\mu\}_{x}$, we present $x$ as function of $\{\mu\}_{x}$ :

$$
x=\sqrt{\frac{3 q\left[\left(\{\mu\}_{x}\right)^{3}-q \tilde{m}^{2}\right]}{2\left(\{\mu\}_{x}\right)^{8}}} .
$$

Thus, the solution will be given by Eq. (55) in the segment if $\left(d \mu_{x} / d x\right) \neq 0$ and constant $\mu_{x}$ in the other segments. In principle, this would allow for a numerical solution. One could actually solve the equation near the transition line using the method in [46]. The situation is completely different compared to that of the $|\psi|^{2}$ disorder. In the present case, a stable RSB solution exists. We will turn first however to the replica symmetric solutions and determine their region of stability. In the unstable region of the replica symmetric solutions, the RSB solution of Eq. (55) will be the relevant one.

\subsection{Two Replica Symmetric Solutions}

\subsubsection{Solutions}

Here, we briefly repeat the steps leading to the RS solutions for the $|\psi|^{2}$ disorder omitting details. The saddle point equations Eq. (25) for the RS matrices $m_{x}=M$ are:

$$
\begin{array}{r}
-\varepsilon^{-2}+\left(a_{T}+4 \widetilde{m}\right)-q\left[5 \widetilde{m}^{3}-M^{3}-2 M^{2} \widetilde{m}-2 \widetilde{m}^{2} M\right]=0 \\
M\left[2 \varepsilon^{-3}-q\left(M^{2}+2 \widetilde{m}^{2}\right)\right]=0
\end{array}
$$

where $\varepsilon \equiv \widetilde{m}-M$. Energy of such a solution is given by

$$
\begin{aligned}
\frac{f}{2}= & \varepsilon^{-1}-\varepsilon^{-2} M+a_{T} \tilde{m}+2 \widetilde{m}^{2} \\
& -\frac{q}{4}\left(5 \widetilde{m}^{2}-M^{2}\right)\left(\widetilde{m}^{2}+M^{2}\right) .
\end{aligned}
$$

For the diagonal solution $M=0, \varepsilon=\widetilde{m}$, and the first equation takes a form:

$$
-\widetilde{m}^{-2}+a_{T}+4 \widetilde{m}-5 q \tilde{m}^{3}=0 .
$$

The nondiagonal solution in the present case is more complicated, but the condition determining the transition line between the two (equivalently the appearance of the nonvanishing EA order parameter) is still very simple: $\varepsilon=q^{-1 / 5}$ as $M=0$ on the line. Along the line, the scaled temperature is:

$$
a_{T}^{d}=2\left(3 q^{2 / 5}-2 q^{-1 / 5}\right) .
$$

It is still the third-order transition line similar to the $|\psi|^{2}$ disorder case, and one has zero modes in NEA sector, while no such modes exist in the $M=0$ phase. The stability analysis with respect to configurations which are replica symmetric however gives completely different results compared to that of the $|\psi|^{2}$ disorder.

\subsubsection{Stability Rgion of the Rs Solutions}

The Hessian now has several additional terms

$$
\begin{aligned}
& H_{(a b)(c d)} \equiv \frac{n}{2} \frac{\delta^{2} f}{\delta m_{a b} \delta m_{c d}}=\left(m^{-2}\right)_{a c}\left(m^{-1}\right)_{a b} \\
& +\left(m^{-1}\right)_{a c}\left(m^{-2}\right)_{a b}+4 \delta_{a b c d} \\
& -q\left(\begin{array}{c}
\frac{3}{2} \delta_{a c} \delta_{b d} Q_{a b}^{2}+\frac{1}{2}\left(\delta_{a c} \delta_{b d} Q_{b a}^{2}+2 \delta_{a d} \delta_{b c} Q_{a b} Q_{b a}\right) \\
2\left(\delta_{a c} \delta_{b d} Q_{a a} Q_{b b}+\delta_{b c d} Q_{a b} Q_{a a}+\delta_{a c d} Q_{a b} Q_{b b}\right) \\
+\delta_{a b}\left[\delta_{c d}\left(Q_{a c}^{2}+Q_{c a}^{2}\right)+2\left(\delta_{a c} Q_{a d} Q_{d d}+\delta_{a d} Q_{c a} Q_{c c}\right)\right]
\end{array}\right)
\end{aligned}
$$

For replica symmetric solutions $Q_{a b}=m_{a b}=$ $\varepsilon \delta_{a b}+M$, the Hessian can be represented as

$H=c_{+} I_{+}+c_{-} I_{-}+c_{U} U+c_{V} V+c_{J} J+c_{K} K+c_{N} N$,

where new operators $I_{ \pm}, K, N$ are defined as

$$
\begin{aligned}
I_{ \pm} & \equiv \frac{1}{2}\left(\delta_{a c} \delta_{b d} \pm \delta_{a d} \delta_{b c}\right) ; \quad K \equiv \delta_{a b} \delta_{c d} ; \\
N & =\delta_{a b c}+\delta_{a b d}+\delta_{a c d}+\delta_{b c d} .
\end{aligned}
$$

The coefficients are

$$
\begin{aligned}
& c_{+}=2 \varepsilon^{-3}-q\left(3 M^{2}+2 \widetilde{m}^{2}\right) ; \\
& c_{-}=2 \varepsilon^{-3}-q\left(M^{2}+2 \widetilde{m}^{2}\right) ; \\
& c_{U}=4 M^{2} \varepsilon^{-5} ; \quad c_{V}=-3 M \varepsilon^{-4} ; \\
& c_{J}=4-q\left[5\left(\widetilde{m}^{2}-M^{2}\right)+8 \widetilde{m}(\widetilde{m}-M)\right] ; \\
& c_{K}=-2 q M^{2} ; \quad c_{N}=-2 q \tilde{m} M .
\end{aligned}
$$

Generally, the Hessian have four different eigenvalues [54]:

$$
\begin{aligned}
\lambda^{(1,2)} & =c_{+}+\frac{1}{2}\left(c_{J}+4 c_{N} \pm \sqrt{c_{J}\left(c_{J}+8 c_{V}+8 c_{N}\right)}\right) ; \\
\lambda^{(3)} & =c_{+}, \lambda^{(4)}=c_{-}
\end{aligned}
$$

Note that there are new matrices like $I_{+}, I_{-}$when $q \neq 0$. In the case of $q=0, c_{+}=c_{-}$, so that only 
operator $I=I_{+}+I_{-}$appears in this case. Actually, there is $\lambda^{(4)}$, which is the eigenvalue of Hessian on the antisymmetric matrices. However, $\lambda^{(4)}=c_{-} \geq 0$ is always hold on the RS solutions so that it can be ignored in determining the instabilities of those RS solutions. Since the stability analysis is quite complicated, we divide it into several stages of increasing complexity.

\subsubsection{Stability of the States on the Diagonal-Off Diagonal "Transition" Line}

The easiest way to see that the RS solutions can be unstable is to look first at the transition line $a_{T}^{d}$, Eq. (60). On the transition line, one has

$$
c_{ \pm}=c_{U}=c_{V}=c_{N}=0 ; \quad c_{J}=4-13 q^{3 / 5} ;
$$

and the eigenvalues simplify to

$$
\lambda^{(3)}=0 ; \quad \lambda^{(1,2)}=4-13 q^{3 / 5} .
$$

Therefore, it is unstable for $q>q^{t}$

$$
q^{t}=\left(\frac{4}{13}\right)^{5 / 3}
$$

marginally stable at a single point

$$
a_{T}^{t}=-\frac{28}{13}\left(\frac{13}{4}\right)^{1 / 3} \approx-3.2
$$

and stable for $q<q^{t}$. We studied numerically the stability on both sides of this line, see Fig. 2. The diagonal (liquid) solution is stable below the line $\left(a_{T}^{d}=\right.$ $\left.2\left(3 q^{2 / 5}-2 q^{-1 / 5}\right)\right)$ for $q>q^{t}$. The line when $q>q^{t}$, the phase transition line (liquid to glass) is changed to a different line which will be discussed in the next section.

\subsubsection{Stability of the Diagonal Solution}

Equation for $\widetilde{m}$, coefficients in Hessian, and eigenvalues are:

$$
\begin{gathered}
-\widetilde{m}^{-2}+a_{T}+4 \widetilde{m}-5 q \tilde{m}^{3}=0 ; \\
c_{+}=2 \widetilde{m}^{-3}-2 q \tilde{m}^{2} ; \quad c_{J}=4-13 q \tilde{m}^{2} ; \\
\lambda^{(1,3)}=c_{+} ; \quad \lambda^{(2)}=c_{+}+c_{J}=4+2 \tilde{m}^{-3}-15 q \tilde{m}^{2} .
\end{gathered}
$$

While $\lambda^{(1)}$ is positive, $\lambda^{(2)}$ is positive only below the line defined parametrically via

$$
a_{T}=\frac{5-8 \widetilde{m}^{3}}{3 \widetilde{m}^{2}} ; \quad q=\frac{4 \widetilde{m}^{3}+2}{15 \widetilde{m}^{5}}
$$

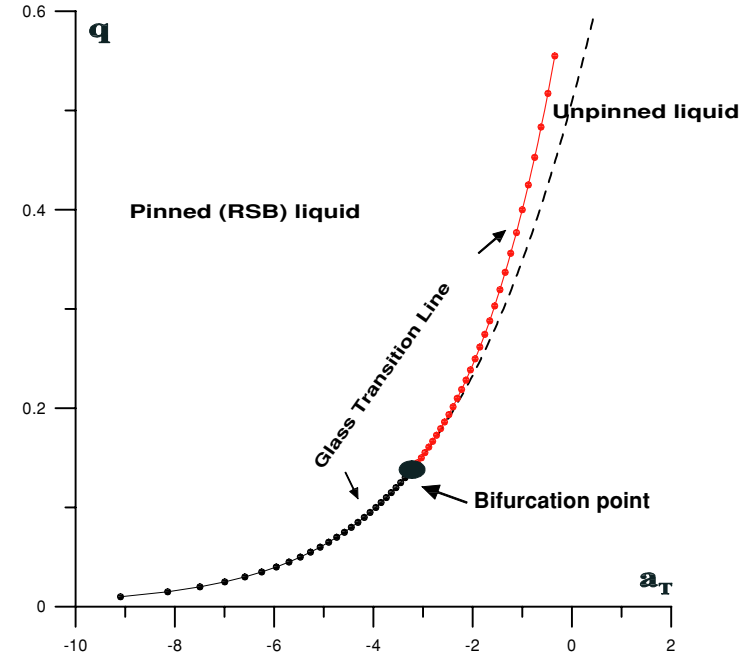

Fig. 2. Phase diagram with the $|\psi|^{4}$ disorder only in the $a_{T}-q$ plane. The dotted line marks the RSB glass transition. The upper part above the "tri-critical" point of this line is given by Eq. (72), while the lower part below the "tri-critical" point of the line is given by Eq. (60). The dashed line is also given by Eq. (60) but does not correspond to a phase transition line. It is just a bifurcation line between two replica symmetric (the diagonal and the off diagonal) states.

marked by dotted line in Fig. 2, and this line is the phase transition line (liquid to glass) when $q>q^{t}$. The former diagonal-off diagonal line above the tricritical point is not a phase transition and is left as a light dashed line to show that slope of the line below tri-critical point and that of the real transition line is different. It turns out that the line of Eq. (72) is a transition line into a RSB state, namely the irreversibility line.

\subsubsection{Stability of the Off Diagonal Solution}

The equations take a form:

$$
\begin{aligned}
& -\varepsilon^{-2}+a_{T}+4 \widetilde{m}-q\left[5 \tilde{m}^{3}-M^{3}-2 M^{2} \widetilde{m}\right. \\
& \left.-2 \widetilde{m}^{2} M\right]=0 \\
& 2 \varepsilon^{-3}-q\left(M^{2}+2 \widetilde{m}^{2}\right)=0 .
\end{aligned}
$$

The coefficients in the expansion of Hessian are: Unlike the case of the $|\psi|^{2}$ disorder, $\lambda^{(1)}<0$ for each such a solution. Therefore, the diagonal state directly goes over into a RSB glass state. It follows however two lines. Equation (72) above the tri-critical point and Eq. (60) below it, see Fig. 2. 


\section{GENERAL CASE RSB}

\subsection{General Hierarchical Gaussian Variational Ansatz}

The free energy

$$
\begin{aligned}
\frac{f}{2 n}= & \sum_{a}\left\{\left(m^{-1}\right)_{a a}+a_{T} m_{a a}+2\left(m_{a a}\right)^{2}\right\} \\
& -\sum_{a, b}\left\{2 r m_{a b}^{2}-\frac{q}{4}\left(m_{a b}^{4}+4 m_{a a} m_{b b} m_{a b}^{2}\right)\right\}
\end{aligned}
$$

leads on the replica symmetric sector to the following equations:

$$
\begin{aligned}
& -\varepsilon^{-2}+a_{T}+4 \widetilde{m}-2 r \varepsilon-q\left[5 \widetilde{m}^{3}-M^{3}\right. \\
& \left.-2 M^{2} \widetilde{m}-2 \widetilde{m}^{2} M\right]=0, \\
& M\left[2 \varepsilon^{-3}-2 r-q\left(M^{2}+2 \widetilde{m}^{2}\right)\right]=0 .
\end{aligned}
$$

For the diagonal solution $M=0$, one obtains

$$
-\widetilde{m}^{-2}+a_{T}+4 \widetilde{m}-2 r \tilde{m}-5 q \widetilde{m}^{3}=0 .
$$

The off diagonal solution on the bifurcation line obeys

$$
\tilde{m}^{-3}-r-q \tilde{m}^{2}=0 .
$$

Hessian for the general RS solution takes a form of Eq. (62) with coefficients

$$
\begin{aligned}
& c_{+}=2 \varepsilon^{-3}-2 r-q\left(3 M^{2}+2 \widetilde{m}^{2}\right) ; \\
& c_{-}=2 \varepsilon^{-3}-2 r-q\left(M^{2}+2 \widetilde{m}^{2}\right) ; \\
& c_{U}=4 M^{2} \varepsilon^{-5} ; \quad c_{V}=-3 M \varepsilon^{-4} ; \\
& c_{K}=-2 q M^{2} ; \\
& c_{N}=-2 q \tilde{m} M ; \\
& c_{J}=4-q\left[5\left(\tilde{m}^{2}-M^{2}\right)+8 \tilde{m}(\tilde{m}-M)\right] .
\end{aligned}
$$

On the bifurcation line, it simplifies:

$$
\begin{aligned}
& c_{ \pm}=2 \widetilde{m}^{-3}-2 r-2 q \widetilde{m}^{2} ; \\
& c_{U}=c_{V}=c_{K}=c_{N}=0 ; \quad c_{J}=4-13 q \widetilde{m}^{2} .
\end{aligned}
$$

The eigenvalues are

$$
\lambda^{(1,2)}=c_{+} ; \quad \lambda^{(3)}=c_{+}+c_{J} .
$$

Therefore, the Hessian vanishes $\lambda^{(1,2)}=\lambda^{(3)}=0$ for the tri-critical (branch) point defined by

$$
r=\left(\frac{13 q}{4}\right)^{2 / 3}-\frac{4}{13} \text {. }
$$

6.1.1.1. Stability of the Diagonal Solution. In this case, Hessian and eigenvalues are:

$$
\begin{aligned}
c_{ \pm} & =2 \widetilde{m}^{-3}-2 r-2 q \widetilde{m}^{2} ; \\
c_{J} & =4-13 q \widetilde{m}^{2} ; \\
\lambda^{(1,2)} & =c_{ \pm} ; \\
\lambda^{(3)} & =c_{ \pm}+c_{J}=4+2 \widetilde{m}^{-3}-2 r-15 q \widetilde{m}^{2} .
\end{aligned}
$$

Below the tri-critical point, we solve equation $\lambda^{(1)}=$ 0 perturbatively in $q$ :

$$
\widetilde{m}=r^{-1 / 3}\left(1-\frac{q}{3} r^{5 / 3}\right)+O\left(q^{2}\right)
$$

and substitute $\widetilde{m}$ into Eq. (77) to determine the "weak disorder" part of the glass transition line:

$$
a_{T}^{g 1}=-\frac{4-r}{r^{1 / 3}}+\left(\frac{5}{r}+\frac{4 r^{4 / 3}}{3}\right) q+O\left(q^{2}\right) .
$$

Above the tri-critical point, namely for larger disorder, one solves the equation $\lambda^{(3)}=0$ perturbatively in $q$ around the tri-critical point of Eq. (82),

$$
\begin{aligned}
q^{t}(r) & =\frac{4}{13}\left(r+\frac{4}{13}\right)^{3 / 2}: \\
\widetilde{m} & =\left(r+\frac{4}{13}\right)^{-1 / 3}\left(1-\frac{10}{24+13 r} \Delta\right)+O\left(\Delta^{2}\right) \\
\Delta & =\frac{q}{q^{t}(r)}-1 .
\end{aligned}
$$

The NEA RS solution is unstable everywhere as $c_{+}<$ $0\left(c_{+}<c_{-}=0\right)$. We, therefore, obtain the glass transition line with RSB in the general case. To compare it with experiment, one has to specify phenomenologically the precise dependence of the GL model parameters on temperature.

\section{TWO-DIMENSIONAL GINZBURG-LANDAU THEORY}

Similarly, the disordered GL is

$$
\begin{aligned}
F= & L_{z} \int d^{2} x \frac{\hbar^{2}}{2 m^{*}}(1+U(x))\left(-\psi^{*} \vec{D}^{2} \psi\right) \\
& +a^{\prime}(1+W(x))|\psi|^{2}+\frac{b^{\prime}}{2}(1+V(x))|\psi|^{4} \\
& +\frac{(B-H)^{2}}{8 \pi},
\end{aligned}
$$


where $L_{z}$ is the layer distance and this model is obtained from the reduction of three-dimensional model, and the disorders are introduced via

$$
\begin{aligned}
m^{*-1} & \rightarrow m^{*-1}(1+U(x)) ; \\
\overline{U(x) U(y)} & =P \delta(x-y) .
\end{aligned}
$$

The other two parameters in the GL equations are $\alpha$ and $b^{\prime}$. The coefficient of the quadratic term is called $\delta T$ disorder, since it describes a local deviation of the critical temperature. Introducing a random component in $|\psi|^{2}$ term:

$$
\begin{gathered}
a^{\prime} \rightarrow a^{\prime}(1+W(x)) ; \quad \overline{W(x) W(y)}=R^{\prime} \delta(x-y) . \\
b^{\prime} \rightarrow b^{\prime}(1+V(x)) ; \quad \overline{V(x) V(y)}=Q \delta(x-y) .
\end{gathered}
$$

In unconventional superconductors, even without disorder, the phenomenological GL has not been reliably derived microscopically. The coefficients and their inhomogeneitics therefore should be considered as phenomenological parameters to be fitted to experiments. We assume that $U, R$, and $Q$ have weak dependencies on field and temperature. The assumption of the weak temperature and field dependence of the disorder strengths $U, R$, and $Q$, as that of any parameter in the GL approach, should be derived in principal from a microscopic theory assuming random chemical potential or should be justified by fitting to experiments. Assuming, for simplicity, the white noise distribution

$$
p[U, W, V]=\exp \left[-\int_{x} \frac{U(x)^{2}}{2 P}+\frac{W(x)^{2}}{2 R^{\prime}}+\frac{V(x)^{2}}{2 Q}\right]
$$

for random components, the free energy of superconductor after averaging over the disorder is

$$
\begin{aligned}
\bar{F}= & -\frac{T}{\operatorname{norm}} \int_{U, W, V} p[U, W, V] \log \left[\int_{\psi} \exp [-g[\psi]\right. \\
& \left.\left.-f_{\text {dis }}[U, W, V, \psi]\right]\right] ; \\
g= & G / T, \quad f_{\text {dis }}[U, W, V, \psi]=\frac{L_{z}}{T} \int_{x} \frac{\hbar^{2}}{2 m_{\perp}^{*}} U(x)|\vec{D} \psi|^{2} \\
& +a^{\prime} W(x)|\psi|^{2}+\frac{1}{2} V(x)|\psi|^{4},
\end{aligned}
$$

where norm $=f_{U, W, V} p[U, W, V]$ is a normalization factor.
We rescale the length and field as in three dimensions:

$$
\begin{gathered}
g=\frac{F}{T}=\frac{1}{\omega} \int d^{2} x\left[\frac{1}{2}\left(-\psi^{*} \vec{D}^{2} \psi\right)-\frac{1-t}{2}|\psi|^{2}\right. \\
\left.+\frac{1}{2}|\psi|^{4}+\frac{\kappa^{2}(b-h)^{2}}{4}\right] . \\
f_{\text {dis }}[U, W, V, \psi]=\frac{1}{\omega} \int d^{2} x\left[\frac{U(x)}{2}\left(-\psi^{2} \vec{D}^{2} \psi\right)\right. \\
\left.-\frac{1-t}{2} W(x)|\psi|^{2}+\frac{1}{2} V(x)|\psi|^{4}\right] \\
p[U, W, V]=\exp \left[-\xi^{2} \int_{x} \frac{U(x)^{2}}{2 P}+\frac{W(x)^{2}}{2 R^{\prime}}+\frac{V(x)^{2}}{2 Q}\right]
\end{gathered}
$$

where the two-dimensional Ginzburg number

$$
G i=\left(\frac{8 \pi e^{2} \lambda^{2} T_{\mathrm{c}}}{e^{2} h^{2} L_{z}}\right)^{2}
$$

and

$$
\omega=\sqrt{2 G i} \pi^{2} .
$$

The model without disorder then simplifies due to the LLL constraint,

$$
-\frac{D^{2}}{2} \psi=\frac{h}{2} \psi
$$

to:

$$
\begin{aligned}
g \equiv \frac{F}{T}= & \frac{1}{\omega} \int d^{2} x\left[-\frac{1-t-b}{2}|\psi|^{2}\right. \\
& \left.+\frac{1}{2}|\psi|^{4}+\frac{\kappa^{2}(b-h)^{2}}{r}\right] .
\end{aligned}
$$

This reduced model exhibits the LLL scaling. Rescaling again $x \rightarrow x / \sqrt{b}, y \rightarrow y / \sqrt{b}$, and $|\psi|^{2} \rightarrow$ $|\psi|^{2} \sqrt{b \omega / 4 \pi}$, one obtains

$g=\frac{1}{4 \pi} \int d^{2} x\left[a_{T}|\psi|^{2}+\frac{1}{2}|\psi|^{4}+\left(\frac{b \omega}{4 \pi}\right)^{-1} \frac{\kappa^{2}(b-h)^{2}}{4}\right]$, where the two-dimensional LLL-reduced temperature

$$
a_{T} \equiv-\sqrt{\frac{4 \pi}{b \omega}} \frac{1-t-b}{2}
$$

is the only parameter in the theory [42,50]. In total, we have done the rescaling

$$
\begin{gathered}
|\psi|^{2} \rightarrow|\psi|^{2}\left(\frac{2 \alpha T_{\mathrm{c}}}{b^{\prime}}\right)\left(\sqrt{\frac{b \omega}{4 \pi}}\right), \\
x \rightarrow \xi x / \sqrt{b}, y \rightarrow \xi y / \sqrt{b} .
\end{gathered}
$$


Including the disorder, one obtains:

$f_{\text {dis }}[U, W, V, \psi]=\frac{1}{4 \pi} \int d^{2} x\left[\Omega(x)|\psi|^{2}+\frac{1}{2} V(x)|\psi|^{4}\right]$

and the disorder correlations are given as

$$
\begin{aligned}
p[\Omega, V] & =\bar{p}(\Omega, V)=\exp \left[-\int_{x} \frac{\Omega(x)^{2}}{2 r^{\prime}}+\frac{V(x)^{2}}{2 q^{\prime}}\right] \\
q^{\prime} & =Q \frac{b}{\xi^{2}}, \quad r^{\prime}=\frac{\pi}{\xi^{2} \omega}\left[b^{2} P+(1-t)^{2} R^{\prime}\right] .
\end{aligned}
$$

Again, as in three dimension, using the replica trick and expanding $\psi(x)$ on the basis of the LLL wavefunctions with quasimomenta $k, \psi_{a}(x) \propto$ $\int d k_{\varphi k}(x) \psi_{a}(k)$, the gaussian effective free energy can be expressed via the variational matrix parameters $m_{a b}(k)=\left\langle\psi_{a}^{*}(k) \psi_{b}(-k)\right\rangle$,

$$
\begin{aligned}
\bar{G} \propto & \sum_{a}\left[-\log m+a_{T} m-r m^{2}\right. \\
& \left.-q(\tilde{m})^{2} m^{2}\right]_{a a}-\frac{q}{4} \sum_{a, b} m_{a b}^{4},
\end{aligned}
$$

where the dimensionless parameters are: the LLL temperature

$$
a_{T}=-(b t \pi \sqrt{2 G i})^{-1 / 2}(1-t-b),
$$

disorder variances

$$
\begin{aligned}
& r=\frac{b^{2} P+(1-t)^{2} R^{\prime}}{4 \pi^{2} \xi^{2} \sqrt{2 G i}}, \\
& q=\frac{4 b Q}{\pi \xi^{2}} .
\end{aligned}
$$

In the homogeneous phase, the matrix $m(k)$ in two dimensional case is independent of $k$ within gaussian approximation (the Landau degeneracy), while in three-dimensional case, there is the $k_{z}^{2}$ contribution to inverse propagator, $\tilde{m}$ is the diagonal matrix element. The glass state is characterized by the loss of ergodicity and reversibility with respect to dynamic processes. This is expressed, formally, by spontaneous breaking of the replica permutation symmetry (RSB). It was shown by Parisi, in the context of the spin glass theory, that the correct solution for the theory of this type is given by the subclass of the matrices $m_{a b}$ which has a hierarchical structure and can be parameterized by the Parisi function $m(x), 0<x<1$.

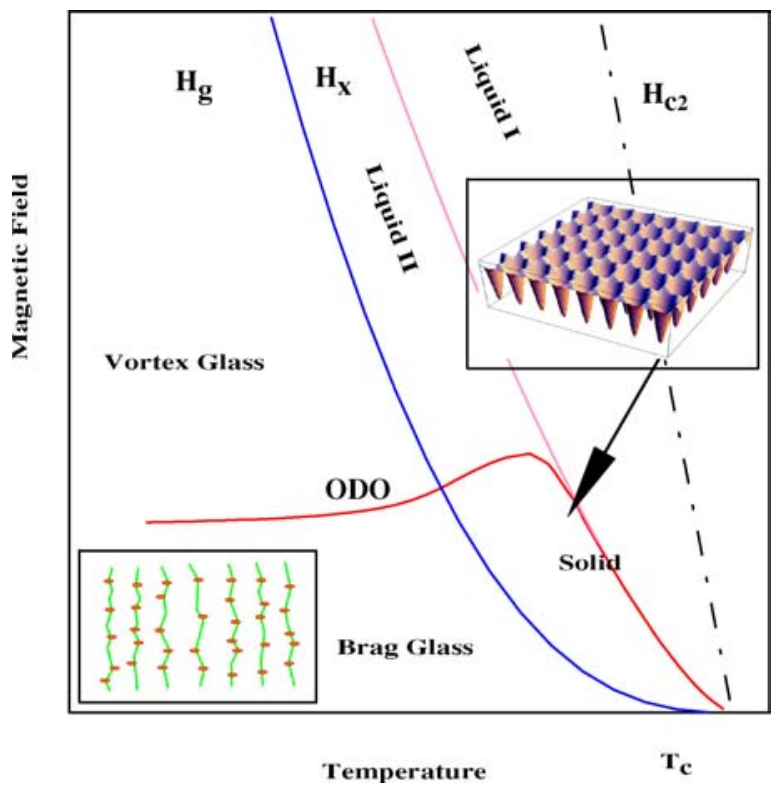

Fig. 3. Generic phase diagram of the vortex matter. The orderdisorder line (red) separates the crystalline phase from the homogenous phase. The glass transition line (blue) separates the glass from the weakly pinned phases, while the pink line is a crossover between two homogeneous phases, locally pinned liquid I and essentially unpinned liquid II. The left inset shows welldefined vortex lines pinned by impurities in Bragg glass region and the right inset shows the distribution of the order parameter in the Abrikosov lattice near the melting line.

In particular, the well-known EA glass order parameter corresponds to $m(x=1)$. The label $x$ reflects the "hierarchy level" and corresponds to the overlap between different "valleys" in the potential landscape [55]. Figure 3 shows the generic phase diagram. We find that in the disordered liquid (domain to the right of the irreversibility line in Fig. 3) the replica symmetric solution is stable, while in the glassy phase (the left side of the line) a nontrivial Parisi function describes a continuous RSB. The irreversibility line for small $q$ is given by

$$
a_{T}^{g}=2 \sqrt{2}\left(r^{1 / 2}-r^{-1 / 2}\right)+\frac{2+5 r}{2 \sqrt{2} r^{5 / 2}} q+O\left(q^{2}\right),
$$

\section{CONNECTION WITH THE EXTREME ORDER STATISTICS AND DYNAMICS}

Now we make connection to the vortex dynamics. Bouchaud and Mezard [56] have demonstrated that for several models of glasses, in particular, the 
random energy model, the one step RSB solution corresponds to the extreme-value statistics for the energy barriers. We remind now, reiterating results of [31], that the extreme-values statistics can be derived for barriers controlling creep vortex motion establishing thus the connection between the replica description of GL functional and phenornenological vortex dynamics. The stationary dynamics of the vortex glass driven by the applied force $F$ is controlled by thermally activated jumps of correlated regions of the vortex elastic manifold over the pinning energy barriers separating different metastable states into neighboring states favored by the applied drive.

\subsection{Dynamics of Clastic Manifolds}

The energy of an elastic manifold driven through a disordered medium can be written as [23,26]

$$
\mathcal{F}=\int_{\mathbf{x}}\left[\frac{C}{2}\left(\frac{\partial \mathbf{u}}{\partial \mathbf{x}}\right)^{2}+V(\mathbf{x}, \mathbf{u})-\mathbf{F} \cdot \mathbf{u}\right],
$$

where $\mathbf{u}(\mathbf{x}, t)$ is the $n$-dimensional displacement vector describing long-wavelength deformations of the medium, with $\mathbf{x}$ a $D$-dimensional vector labeling the internal coordinates of the manifold, which in turn is embedded in a $d$-dimensional space. Also, $C$ is an elastic stiffness and $\mathbf{F}$ the external drive. Equation (101) describes, for instance, a vortex line $(D=1)$ in two $(n=1, d=2)$ and three $(n=2, d=3)$ dimensions, or a vortex lattice in a superconducting film $(n=2, D=2, d=2)$ or in bulk $(n=2, D=3$, $d=3$ ). The disorder is described by a random potential $V(\mathbf{x}, \mathbf{u})$ of range $\xi$, zero mean, $\langle V\rangle=0$, and variance $\Delta_{0}$,

$$
\left\langle V(\mathbf{x}, \mathbf{u}) V\left(\mathbf{x}^{\prime}, \mathbf{u}^{\prime}\right)\right\rangle=\Delta_{0}^{2} \delta^{D}\left(\mathbf{x}-\mathbf{x}^{\prime}\right) g\left(\left|\mathbf{u}-\mathbf{u}^{\prime}\right| / \xi\right),
$$

with $g(s)$ a dimensionless function that decays rapidly for $s>1$.

For simplicity, we will refer specifically to the case of a directed elastic string in $d=2$ or $d=$ 3 dimensions, a generalization onto arbitrarily dimension elastic system is straightforward. This is the problem of a directed polymer in a random medium whose static properties have been studied extensively in recent years. In $d=3$ and in the absence of external drive, the elastic string is in a disorder-dominated pinned state for all temperatures and pinning strengths. In this pinned state (zerotemperature, strong-disorder fixed point), the string adjusts to the random landscape and traverses the medium along an optimal path determined by balancing the elastic and the pinning energies. This optimal path is characterized by its roughness, defined as the mean square displacement for a deformation that extends over a linear size $L$,

$$
w(L)=\langle[\mathbf{u}(\mathbf{x}+\mathbf{L})-\mathbf{u}(\mathbf{x})]\rangle^{1 / 2},
$$

where $\langle\cdots\rangle$ denotes the average over both thermal fluctuations and the disorder potential. If the roughness $w(L)$ is less than the range $\xi$ of the random potential, the corresponding segment of size $L$ is pinned coherently by disorder. This defines the collective pinning length $L_{\mathrm{c}}$ above which the roughness exceeds the range $\xi$ of fluctuations in the random potential, as $w\left(L_{\mathrm{c}}\right)=\xi$. The collective pinning length $L_{\mathrm{c}}$ can be estimated via dimensional analysis. The elastic energy cost of a deformation where the elastic manifold is displaced a distance $w$ over a length $L$ is,

$$
\left\langle\left(\Delta E_{c}\right)^{2}\right\rangle^{1 / 2} \sim C \frac{w^{2}}{L^{2}} L^{D} .
$$

The pinning length $L_{c}$ is determined by balancing the elastic energy cost of a deformation with $w \sim \xi$ against the corresponding gain in pinning energy,

$$
\left\langle E_{\mathrm{p}}^{2}\right\rangle^{1 / 2} \sim \Delta_{0} L^{D}
$$

where $E_{\mathrm{p}}=\int_{x} V(\mathbf{x}, \mathbf{u})$ denotes the fluctuating pinning energy, with the result

$$
L_{c}=\left(\frac{C \xi^{2}}{\Delta_{0}}\right)^{2 /(4-D)} .
$$

The corresponding collective pinning energy is given by Eq. (105) for $L=L_{c}$,

$$
U_{c}=\Delta_{0} L_{c}^{D} .
$$

The collective pinning energy represents the minimum energy barrier between neighboring metastable configurations of the pinned string. Similarly, $L_{c}$ represents the shortest length scale over which nonvanishing energy barriers between metastable states exist for $F=0$.

At distances $L \gg L_{c}$, it is found numerically that the roughness scales as $w(L) \sim L^{\zeta}$, with $\zeta<1$ the roughness exponent. For an elastic string in three dimension, $\zeta \sim 0.6$, a value larger than the corresponding value for a string in the presence of thermal fluctuations, but no disorder. The free energy differences between two metastable configurations on scale $L>$ $L_{c}$ are distributed with zero mean and variance given 
by Eq. (104) for $w \sim L^{\zeta}$,

$$
\left\langle[\Delta \mathcal{F}(L)]^{2}\right\rangle^{1 / 2} \sim C L^{\theta},
$$

with $\theta=2 \zeta+D-2$. The barriers separating such neighboring metastable states are distributed with mean

$$
U(L)=\langle\hat{U}(L)\rangle \sim \Delta_{0} L^{\psi}
$$

and variance $\left\langle[\Delta \hat{U}(L)]^{2}\right\rangle^{1 / 2} \sim \Delta_{0} L^{\psi}$ (with likely logarithmic corrections) that also grows with the size of the excitation. If the motion occurs via continuous deformation of an elastic medium, without the introduction of topological defects, one expects $\psi \geq \theta$. Values of $\psi<\theta$ are also possible in principle when the motion is along percolating low-energy channels. In general, it is assumed that $\psi=\theta$. This assumption has been confirmed for the case of a string in $d=2,3$ via a combination of analytical and numerical arguments [30,57]. The question of whether $\psi=\theta$ for all elastic media remains, however, open. When a driving force $F$ is applied, at zero temperature, the string starts to slide when the force $F$ can depin a region of linear size $L_{c}$. This corresponds to the condition $F \xi L_{c}^{D} \sim C \xi^{2} L_{c}^{D-2}$, which yields a threshold for sliding, $F_{c} \sim C \xi^{2} / L_{c}^{2}$. At finite temperatures and for driving forces below $F_{T}$, the manifold can cross from one local minimum to another via thermal activation over the free energy barriers $U(L) \sim \Delta_{0} L^{\psi}$. The low-lying excitations consist of displacements of a portion of string of length $L$ by a transverse distance $w$ in the direction of the applied force. Such excitations can be described as directed vortex loops (Fig. 4), when viewed with respect to the ground state configuration. Dynamics then occurs via the nucleation and growth of vortex loops and scaling arguments can be used to infer the response to the applied force. The loop has free energy $F \sim C L^{\theta}$. An applied force can create vortex loops of typical size $L_{F}$, determined by balancing the energy of the loop, $C L^{\theta}$, against the energy provided by the external force, $F L^{\zeta+D}$. This gives $L_{F}=(F / C)^{1 /(\zeta-2)}$. To obtain these loop excitations from the ground state, the vortex line has to pass over a free energy barrier $\mathcal{U}(L) \sim \Delta_{0} L^{\psi}$. Motion then occurs via thermal nucleation of loops of size $L_{F}$ at a rate proportional to $e^{-\mathcal{U}\left(L_{F}\right) / k_{B} T}$. The resulting mean velocity of the medium is given by

$$
v \propto \exp \left[-\frac{U_{c}}{T}\left(\frac{F_{c}}{F}\right)^{\mu}\right],
$$

with $\mu=\psi /(2-\zeta)$. If one assumes that $\psi=\zeta$, then $\mu=(2 \zeta+D-2) /(2-\zeta)$.

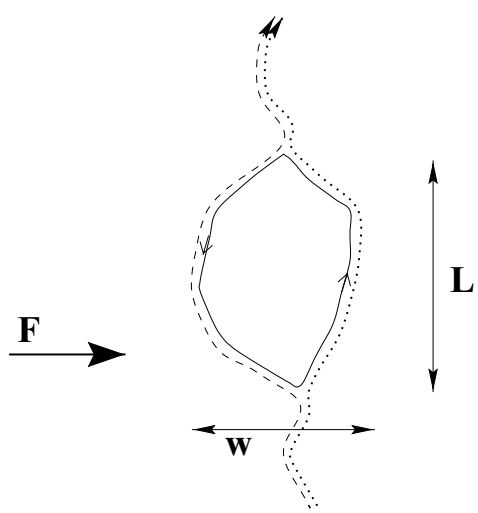

Fig. 4. The low-temperature dynamics is dominated by thermally activated transitions between "neighboring" metastable states (i.e., ones with free energy separation $\triangle E \ll k_{B} T$ ) separated by large barriers $\left(U \gg k_{B} T\right)$.

To summarize, the stationary dynamics of the vortex glass driven by the applied force $F$ is controlled by thermally activated jumps of correlated regions of the vortex elastic manifold over the pinning energy barriers separating different metastable states into neighboring states favored by the applied drive. The pinning length, $L_{\mathrm{c}}$, is the smallest scale on which barriers between metastable states exist at $F=0$. The minimum average energy barrier between adjacent metastable positions of a pinned segment $L_{c}$ is $U_{c}=C \xi^{2} / L_{c}^{D-2}$, sets the energy scale for the vortex system, and $F_{c} \sim U_{c} / \xi$ ( $\xi$ is the scale of the random potential) is the threshold pinning force. In the thermally activated (creep) regime, the mean velocity is highly nonlinear [23]: $v=\exp [-\mathcal{U}(F) / T]$, where $\mathcal{U}(F) \sim U_{c}\left(F_{c} / F\right)^{\mu}$ is the energy barrier separating low lying metastable states of the vortex array, $\mu=(2 \zeta+D-2) /(2-\zeta)$, and $\zeta$ is the roughening exponent. Creep dynamics is the result of all the possible thermally activated jumps averaged with the appropriate distribution function of energy barriers. The important note is that the length $L_{F}$ sets the maximum spatial scale at which pinning still exists; accordingly, $\mathcal{U}(F)$ is the largest possible creep barrier corresponding to this length.

\subsection{Extreme Events Statistics and the Stability Postulate}

Equation (110) is viewed as an operational definition of the glassy state as a state where the linear response to the infinitesimal force is absent, reflecting the hierarchical nature of the glass configurational 
space and, as a result, the multiple spatial/temporal scales present. The renormalization group (RG) picture provides a natural framework for discussing equilibrium properties and relaxation in systems characterized by many length and time scales, and indeed, recently the result (110) was re-derived using the refined RG approach [32]. A new insight into the origin of glassy behavior can be derived from combining RG and scaling ideas with the theory of extreme order statistics relating the statistics of energy levels in glassy systems $[31,56,58]$ and that of spatial disorder in random media [59], see also [60].

The idea of combining scaling ideas with extreme statistics goes back to the pioneering works by Fisher and Tippett and of Frechet [61,62], which, having transliterated into a contemporary terminology contain all the basic ideas of the spatial renormalization group approach. The aforementioned authors were to identify the "universal" properties of the distribution functions of the largest (smallest) value of a large set of the independent identically distributed random variables $x_{i}, i=1,2, \ldots, n$. For the sake of completeness, we will give a brief review of the necessary results of the extreme order statistics concepts following [61] (see also [63,64] for a good introduction into the ideas of extreme statistics and stable distributions). Let $F_{1}(x)$ be the probability that any of the random variables $x_{i}$ be less than $x$ and let $f(x)=F_{1}^{\prime}(x) \equiv\left(d F_{1} / d x\right)$ with support in $[a, b]$, be the normalized probability density, henceforth called the initial distribution. The probability that the largest value of the set $\left\{x_{1}, x_{2}, \ldots, x_{n}\right\}$ be less than $x$ is simply the probability that all of the $x_{i}$ 's fall short of $x$. Since the variables are independent, this is given by

$$
P\left(\max _{1 \leq i \leq n}\left\{x_{i}\right\} \leq x\right) \equiv F_{n}(x)=\left[F_{1}(x)\right]^{n} .
$$

If the initial distribution $f(x)$ is known, then the distribution of the extreme values is easily calculated. The questions that raised much interest starting in the early part of the century were whether asymptotic distributions of extreme values of a large sample of independent random variables exist, how quickly the asymptotic forms are approached, and how these could be calculated given little or no knowledge of the initial distribution.

The first derivation of such asymptotes is due to Fisher and Tippett $[61,62]$. Following these authors, we consider a system of size $N$ and divide it in $m$ identical parts, each of size $n$, with $N=n m$. The idea now is to look for self-similarity between the whole system (composed of $N=n m$ units) and each of its $m$ parts when $n$, for fixed $m$. The central assumption made by Fisher and Tippett is that in the limit $m \rightarrow \infty$ the distribution of the largest (or smallest) values in each sample of size $m$ will tend to the same fixed point (provided such a fixed point exists) as the distribution of largest values in the sample of size $\mathrm{nm}$. Using the fact that a linear transformation of the argument $x$ does not change the form of the probability distribution $F_{n}(x)$, as can be seen from Eq. (111), it can be shown that, under rather general assumption for the initial distribution $f(x)$, there exist two sequences $\sigma_{n}$ and $\mu_{n}$, such that

$$
\begin{aligned}
\lim _{n \rightarrow \infty} F_{n}(x) & =\lim _{n \rightarrow \infty} F_{n}\left(\sigma_{n} z+\mu_{n}\right) \\
& =\lim _{n \rightarrow \infty} H_{n}(z)=H(z),
\end{aligned}
$$

where we have defined

$$
H_{n}(z)=F_{n}\left(\sigma_{n} z+\mu_{n}\right)
$$

and $H(z)$ is an asymptotic distribution function.

Fisher and Tippett assumed that the distribution of the largest value in a sample of size $N=n m$ should be the same as the distribution of the largest value in each part of size $n$ when $n \rightarrow \infty$, except for a linear transformation. This assumption is referred to as the stability postulate. The asymptotic distribution for the sample of size $N=n m$ is defined by

$$
\begin{aligned}
\lim _{n \rightarrow \infty} F_{n m}(x) & \\
& =\lim _{n \rightarrow \infty} F_{n m}\left(\sigma_{n m} z+\mu_{n m}\right) \\
& =\lim _{n \rightarrow \infty} H_{m n}(z)=H(z) .
\end{aligned}
$$

We can also write

$$
\begin{aligned}
\lim _{n \rightarrow \infty} F_{n m}(x) & \\
& =\lim _{n \rightarrow \infty} F_{n m}\left(\sigma_{n m z}+\mu_{n m}\right) \\
& =\left[\lim _{n \rightarrow \infty} F_{n}\left(\sigma_{n m z}+\mu_{n m}\right)\right]^{m} \\
& =\left[\lim _{n \rightarrow \infty} H_{n}\left(\frac{\sigma_{n m}}{\sigma_{n}} z+\frac{\mu_{n m}-\mu_{n}}{\sigma_{n}}\right)\right]^{m} \\
& =\left[H\left(a_{m z}+b_{m}\right)\right]^{m},
\end{aligned}
$$

where

$$
\begin{aligned}
& a_{m}=\lim _{n \rightarrow \infty} \frac{\sigma_{n m}}{\sigma_{n}}, \\
& b_{m}=\lim _{n \rightarrow \infty} \frac{\mu_{n m}-\mu_{n}}{\sigma_{n}} .
\end{aligned}
$$


The stability postulate requires that Eqs. (112) and (116) be equal, or

$$
H(z)=\left[H\left(a_{m z}+b_{m}\right)\right]^{m},
$$

which provides a functional equation for the asymptotic probability $H(z)$.

There are three solutions of Eq. (119), depending on the support of the initial distribution. Details of the derivation of the three solutions can be found for instance in [61]. The first one is the Fréchet distribution obtained for probability densities that are unlimited to the right and given by

$$
\begin{aligned}
& H_{F}(z)= \begin{cases}e^{-z^{-\alpha}} & \text { for } z>0 \\
0 & \text { for } z \leq 0\end{cases} \\
& \text { with } \quad a_{m}=m^{1 / \alpha}, b_{m}=0,
\end{aligned}
$$

with $\alpha>0$. The second solution applies for distributions that are unlimited to the left and is known as the Weibull distribution, with

$$
\begin{aligned}
& H_{F}(z)= \begin{cases}1 & \text { for } z \geq 0 \\
e^{-(-z)^{\alpha}} \text { for } z>0\end{cases} \\
& \text { with } \quad a_{m}=(1 / m)^{1 / \alpha}, \quad b_{m}=0,
\end{aligned}
$$

with $\alpha>0$. Finally, the third solution is the FisherTippett distribution, given by

$$
\begin{aligned}
H_{\mathrm{FT}}(z) & =e^{-e^{-z}} \\
\text { with } a_{m} & =1, \quad b_{m}=\ln m .
\end{aligned}
$$

In a modern language, the stability Eq. (119) is the functional equation that determines the fixed point of an RG flow in the space of distribution functions, as discussed recently in [59]. The main assumption is the existence of such a fixed point.

\section{STATISTICS OF ENERGY BARRIERS AND LOW-TEMPERATURE DYNAMICS}

\subsection{Energy Barriers for a Pinned String}

Consider a pinned string of length $L$ as a necklace of $N$ coherently pinned units or beads of length $L_{c}$ (see Fig. 5), with $L=N L_{c}$. A low-lying fluctuation of one of the $i$ th bead corresponds to its displacement by a transverse distance $\xi$ over the elemental energy barrier $U_{i}$, where the $U_{i}$ 's are statistically independent random variables with mean $U_{c}$. For an elastic medium with $D>1$, the description as a network of beads is still applicable and useful,

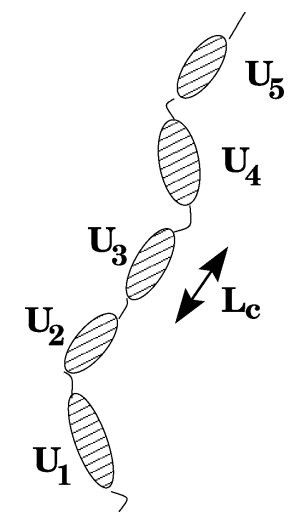

Fig. 5. An elastic string viewed as a sequence of elementary units of size $L_{c}$. The low-lying excitations of the string corresponds to displacements of one unit by a transverse distance $\sim \xi$. The energy barrier that must be overcome for nucleating one such excitation from the ground state is a random variable with mean $U_{c}$.

even though the network no longer has a simple linear topology (see $[58,65])$.

A thermally activated hop of the whole string between the two neighboring metastable states can be described as a result of all possible combinations of all the possible hops (and returns) of the units of the size $L_{c}$ to new metastable positions across the distance $\xi$ and over the energy barrier $U_{i}$. Then the departure of the string segment consisting of, say, $n$, subsequent beads from its original will be controlled by the largest among the fluctuating energy barriers $U_{i}$ corresponding to hops of the elemental units. An important note is in order. Of course, the first hop which occur at the smallest, barrier $U_{k}$ of the chosen segment will renormalize the adjacent barriers due to elastic coupling. This however does not change our consideration. Indeed, let us call denote renormalized barriers as $\bar{U}_{k-1}$ and $\bar{U}_{k+1}$ and include them into the full set of the barriers $\left\{U_{i}\right\}$ describing the segment. Including the results of all the possible elastic renormalizations into $\left\{U_{i}\right\}$, we obtain still the finite set of barriers, the maximal of which will be controlling the departure of the segment from its initial position. Since for the following decimation procedure the opportunity to choose the maximal barrier out of the given set is relevant, we assume that the results of such an elastic renormalization are already taken into account. We thus start with a pinned string segment of length $L$ consisting of a collection of $N=$ $L / L_{c}$ units $L_{c}$. As discussed earlier, thermal activation of this segment is controlled by $\max _{1 \leq i \leq N}\left\{U_{i}\right\}$. The first step consists in combining $n$ units of size $L_{c}$ "in series," in order to define blocks of size $n$. 
The string segment can now be thought of as composed of $m$ blocks, each, in turn, consisting of $n \geq 1$ elementary units $L_{c}$. Each block has size $L_{n}^{(1)}=n L_{c}$ and $L=m L_{n}^{(1)}$. The thermally activated hop of each block of size $L_{n}^{(1)}$ can be described as done above for the hop of the whole string and will therefore be controlled by $\max _{1 \leq i \leq n}\left\{U_{i}\right\}$, for $i=1,2, \ldots, m$. We then repeat this procedure by combining $n$ blocks into $m$ new blocks of size $L_{n}^{(2)}=n L_{n}^{(1)}$, so that the string is given by $L=m L_{n}^{(2)}$. Again, the thermally activated motion of these new blocks is controlled by the largest among the barriers for each block. This procedure allows us to account for all configurations of the pinned segment, by describing it as a topologically linear collection of blocks of all sizes assembled in random order. Due to the self-similarity of the block structure it is natural to assume that the stability postulate will apply at all stages of the renormalization procedure. Following Fisher and Tippett, for large $n$, the probability distribution of the largest barrier in a block of the size $n$ will be identical to the distribution of the largest barrier for the whole string. Thus, the probability that the largest energy barrier controlling the hop of a string segment of the length $L$ is less than $U$ is given by the solution of the functional fixed point Eq. (119), with $z=\left(U / U_{c}-b_{n}\right) / a_{n}$ and all lengths measured in units of $L_{c}$.

As discussed in the previous section, the functional equation has three solutions. We now consider the situations with weak and strong pinning and choose the appropriate solution.

\subsubsection{Weak Pinning}

Let $f(U)$ denote the probability density for each of the elementary random barriers $U_{i}$, with $\left\langle U_{i}\right\rangle=U_{c}$ and $\left\langle U_{i} U_{j}\right\rangle=\delta_{i j} U_{c}^{2}$. For the case of weak pinning, we can model disorder as described by a probability density with support in $[-\infty,+\infty]$. The relevant solution of Eq. (119) is then the Fisher-Tippett solution given by Eq. (122). The probability distribution of the largest energy barriers for a pinned segment of length $L$ is then,

$$
\begin{aligned}
\mathcal{P}_{L}(U) & \sim \exp \left[-e^{-\left[U-U_{\mathrm{c}} \ln \left(L / L_{\mathrm{c}}\right)\right] / U_{\mathrm{c}}}\right] \\
& =\exp \left[-\left(L / L_{\mathrm{c}}\right) e^{-U / U_{\mathrm{c}}}\right] .
\end{aligned}
$$

and the corresponding probability density is given by

$$
p_{L}(U)=\frac{d \mathcal{P}_{L}}{d U} \sim \frac{L}{L_{c}} e^{-U / U_{c}} \exp \left[-\left(L / L_{c}\right) e^{-U / U_{c}}\right] .
$$

The most probable value of the energy barrier corresponds to the maximum of the probability density and therefore scales as $U^{*} \sim U_{c} \ln \left(L / L_{c}\right)$. The distribution function decays rapidly for large arguments and it is asymmetric about its maximum. The mean value of the largest barrier is larger than the most probable value. Finally, the variance of the distribution of largest energy barriers is independent of the length of the string segments and proportional to $U_{c}$.

\subsubsection{Strong Pinning}

Strong disorder, in contrast, may be described by a probability distribution $f(U)$ with bounded support, such as $f(U)=\Theta(U) \Theta\left(U_{m}-U\right)$, with $\Theta(x)$ the Heavyside step function. Here $U_{m}>0$ is the maximum energy barrier for a single pinning site. A single-pinning-site probability distribution in the form of a step function contains a single energy scale, as the maximum barrier $U_{m}$ and the mean barrier $U_{c}$ are simply proportional. More generally, these two energy scales are different and it is important to distinguish them, as the introduction of the new energy scale $U_{m}$ will have important consequences below. For the case of a single-site distribution with finite-support, the relevant solution of Eq. (119) is the Weibull distribution with $\alpha=1$. In terms of the relevant variables, the asymptotic probability that a string segment of length $L$ is separated by a neighboring metastable state by a maximum energy barrier less than $U$ is given by

$$
\begin{aligned}
\mathcal{P}_{L}(U) & =e^{-\left(L / L_{c}\right)\left(U_{m}-U\right) / U_{c}}, \quad U \leq U_{m} \\
& =1, \quad U>U_{m} .
\end{aligned}
$$

The corresponding probability density is

$$
p_{L}(U) \sim \frac{L}{L_{\mathrm{c}}} e^{-\left(L / L_{\mathrm{c}}\right)\left(U_{m}-U\right) / U_{\mathrm{c}}} \Theta\left(U_{m}-U\right) .
$$

In this case, the most probable maximum barrier is trivially the largest barrier $U_{m}$. The variance of the distribution of the largest barriers scales as $L_{c} / L$. In other words, the distribution of the largest barriers becomes sharply peaked about the maximum barrier $U_{m}$ as the sample size increases.

\subsection{Low-Temperature-Driven Dynamics}

We now use the above results for description the finite temperature dynamics of a string driven 
by an external applied force $F \ll F_{T}$, with $F_{T}$ the zero temperature threshold force. To model the dynamics in this region, we consider all string segments of length $L<L_{F}$, corresponding to energy barriers $U<U_{F}=U\left(L_{F}\right)$. These segments form a network of "temporarily pinned" states, while segments of length $L>L_{F}$ are sliding. The slow dynamics in this regime will be described as resulting from the thermally activated motion of the pinned segments with $L<L(F)$. Each of such temporarily pinned segments of length $L$ can be thought of as a collection of units of length $L_{c}$, as discussed above. Its thermally activated dynamics over the rough energy landscape will then be controlled by the largest of the energy barriers controlling the motion of the individual units. The probability distribution of these largest barriers is then given by Eq. (123). At the finite-temperature depinning threshold $(F=0)$ the pinned string segments form a network that has the statistical properties of a percolation cluster near criticality. The cluster size distribution $n_{L}$ is given by $n_{L} \sim\left(L / L_{c}\right)^{-v}$ for large $L$, where $v=1+d / d_{f}$, with $d$ the dimensionality of the medium and $d_{\mathrm{f}}<d$ the fractal dimensionality of the cluster network [65]. Assuming the same distribution for the temporarily pinned segments of length $L<L_{F}$, the global probability density $W(U)$ of the largest barriers is obtained by averaging $P_{L}(U)$ over the cluster-size distribution $n_{L}$,

$$
W(U)=\int_{L_{c}}^{\infty} \frac{d L}{L_{c}} n_{L} p_{L}(U) .
$$

The upper limit of integration may be taken to infinity in Eq. (127) as the integral is in general dominated by small values of $L$. This reflects the fact that elementary hops of segments of length $L_{c}$ greatly outweigh in number hops of larger string segments and therefore end up controlling the barrier statistics. It must, however, be kept in mind that $W(U)$ is the probability density of the largest energy barriers $U$, for $U<U_{F}$. The low-temperature dynamics of the string is then assumed to be dominated by thermally activated hops of the temporarily pinned segments between neighboring metastable states. These hops in turn are controlled by the largest energy barriers, distributed according to Eq. (128). Equivalently, one can define an associated waiting time $\tau=\tau_{0} \exp (U / T)$, with $\tau_{0}$ a microscopic time scale. Later, we obtain the corresponding distribution of waiting times $\Psi(\tau)$, defined by $\Psi(\tau) d \tau=W(U) d U$, for the two relevant cases of weak and strong pinning.

\subsubsection{Weak Pinning}

The integral in Eq. (127), with $\mathcal{P}_{L}(U)$ given by Eq. (124), can be easily evaluated asymptotically by the method of steepest descents, with the result,

$$
W(U) \sim e^{-U(v-1) / U_{c}} .
$$

The distribution $\Psi(\tau)$ of waiting times controlling the hop of string segments of length $L<L_{F}$ decays then as a power law, with

$$
\Psi(\tau) \sim T\left(\tau_{0} / \tau\right)^{1+\alpha},
$$

with $\alpha=(v-1) T / U_{c}<1$. The power-law decay of the waiting time distribution applies for values of $\tau$ up to $\tau_{\max }=\tau_{0} \exp U_{F} / T$. Since $\alpha<1$, the mean waiting time $\langle\tau\rangle$ is controlled by the upper cutoff $\tau_{\max }$, with

$$
\begin{aligned}
\langle\tau\rangle & =\int_{0}^{\tau_{\max }} d \tau \tau \Psi(\tau) \\
& \approx \tau_{0} e^{(1-\alpha) U_{F} / T} .
\end{aligned}
$$

Waiting times larger than $\tau_{\max }$ are excluded by construction in the model, as they correspond to string segments of length $L>L_{F}$ which are sliding. We recover therefore the result that the mean waiting times, which determined the mean drift velocity $v \sim$ $1 /\langle\tau\rangle$, is controlled by the optimal energy barrier $U_{F} \sim F^{-\mu}$.

\subsubsection{Strong Pinning}

The global probability density $W(U)$ is now obtained from Eq. (127), with $p_{L}(U)$ given by Eq. (126). The cluster-size distribution is again a power-law for large $L, n_{L} \sim\left(L / L_{c}\right)^{-s}$, with $2<s<3$. This form for the cluster-size distribution is appropriate for very small driving forces, when the competition between drive and disorder is not sufficient to generate topologically defects in the driven string. At larger driving forces elastic creep of the driven string will be replaced by plastic flow. The cluster-size distribution is expected to become a function of the driving force $F$, yielding additional nonlinearities in the dynamics.

The global probability distribution is given by

$$
\begin{aligned}
W(U) & =\int_{1}^{\infty} d x x^{1-s} e^{-x \epsilon} \Theta(\epsilon) \\
& =\epsilon^{s-2} \Gamma(2-s, \epsilon) \Theta(\epsilon),
\end{aligned}
$$

where $\epsilon=\left(U_{m}-U\right) / U_{c}$ and $\Gamma(-a, \epsilon)$ is the incomplete $\Gamma$-function. Using the asymptotic form of the 
$\Gamma$-function,

$$
\begin{aligned}
\Gamma(-a, \epsilon) & \approx \frac{1}{a \epsilon^{a}}, \quad v \ll 1, \\
& \approx \frac{1}{\epsilon^{1+a}} e^{-\epsilon}, \quad v \gg 1,
\end{aligned}
$$

we find that $W(U)$ is given by

$$
\begin{array}{ll}
W(U) \sim \frac{1}{s-2} \Theta\left(U_{m}-U\right), & \left(U_{m}-U\right) / U_{c} \ll 1, \\
W(U) \sim \frac{1}{\epsilon} e^{-\epsilon} \Theta\left(U_{m}-U\right), & \left(U_{m}-U\right) / U_{c} \gg 1 .
\end{array}
$$

In other words, the probability density of the largest energy barriers is constant for barriers near the maximum barrier $U_{m}$ and decays exponentially as $U$ becomes smaller than $U_{m}$.

The corresponding waiting time distribution is given by

$$
\begin{aligned}
\Psi(\tau) & =\frac{T}{\tau}\left[\frac{T}{U_{c}} \ln \left(\tau_{m} / \tau\right)\right]^{s-2} \Gamma\left(2-s, \frac{T}{U_{c}} \ln \left(\tau_{m} / \tau\right)\right) \\
& \approx\left(\frac{\tau_{m}}{\tau}\right)^{1-\alpha},
\end{aligned}
$$

for $\tau \leq \tau_{m}$, where $\tau_{m}=\tau_{0} \exp U_{m} / T$ and $\alpha \approx T / U_{c}$. The last approximate equality in Eq. (134) applies for $\tau \ll \tau_{m} e^{-U_{\mathrm{c}} / T}=\tau_{0} c^{\left(U_{m}-U_{\epsilon}\right) / T}$.

To summarize, we have found that the distribution function for the relevant activation barriers corresponding to a pinned segment of the size $L$ is given by the extreme value statistics:

$$
P_{L}(E)=\exp \left[-\frac{L}{L_{c}} e^{-E / U_{c}}\right] .
$$

Upon summation over all possible pinned segment sizes with the probability density $p_{L}(E)=$ $d P_{L}(E) / d E$, one arrives at the global exponential distribution of barriers, $W(E) \propto \exp \left(-E / U_{c}\right)$. Since the waiting time $\tau(E)$ for hops between metastable states separated by energy barriers $E<\mathcal{U}(F)$ is thermally activated, the distribution of waiting times scales algebraically at large $\tau$

$$
\Psi(\tau) \propto \frac{1}{\tau^{1+\alpha}},
$$

with $\alpha<1$. This distribution is cut off at $\tau=\tau[\mathcal{U}(F)]$ corresponding to the maximal barrier $\mathcal{U}(F)$, since at $t>\tau[\mathcal{U}(F)]$ the dynamics is no longer thermally activated and string segments of the size $L>L_{c}$ slide freely. Finding the average waiting time $\bar{\tau}$, one recovers the creep motion formula. Therefore, the glassy dynamics relies on the divergence of the average waiting times at low temperatures where $\alpha<$ 1. In the high-temperature region above the depinning temperature $T_{\mathrm{dp}}$, thermal fluctuations renormalize the pinning energy resulting in $\alpha=1$, and the dynamics is only marginally glassy with the power-law $I-V$ characteristics [31] $\nu \propto F^{2+\mu}$. For the threedimensional vortex lattice, we expect the analogous effect to occur at the melting temperature of the pristine lattice (i.e., we expect that $\alpha=1$ at the melting line). Thus, it is the depinning temperature that separates the replica-symmetric and brokenreplica-symmetry regions. We would like to stress here that the observation we made is the connection between the statistics of the barriers controlling vortex motion and the RSB in disordered GL functional. This indicates that the connection we discuss reflect not the mathematical properties of a particular model but rather a very generic physical property of all the systems containing quenched disorder.

Since the energy landscape in an elastic disordered system is characterized by a single energy parameter $U_{c}$, one can speculate that the hierarchy variable $x$ that appears in the GL replica treatment corresponds to the ratio $U / U_{c}$. Accordingly, the distribution of $x$ corresponds to the distribution of the relaxation times. Small $x$ correspond to short relaxation times, while $x \rightarrow 1$ relate to the long times. The microscopic picture for the glass state presented above allows to differentiate between the glass (irreversibility) line and the order-disorder transition line (ODO).

\section{GENERIC PHASE DIAGRAMS AND COMPARISON OF THE THEORETICAL AND EXPERIMENTAL PHASE DIAGRAMS}

\subsection{Generic Phase Diagrams}

Now we turn to physical consequences and discuss the vortex state phase diagram which exhibits three major transitions.

(i) The positional order-disorder line. The firstorder ODO transition signals the loss of the translation and rotation symmetry, and the intensity of the first Bragg peak can be used as an order parameter. The unified first-order ODO line (solid red line in Fig. 3) comprising the melting- and the "second magnetization 
peak" segments, separates the homogeneous and the crystalline phases. The broken symmetry is not directly related to pinning, however the location of the line is sensitive to disorder [16]. Note the twofold role of thermal fluctuations which make quenched disorder destroying the positional order less effective but, at the same time, they themselves destabilize vortex lattice. The low-temperature segment of the ODO line, the second peak line, is disorder dominated and depends weakly on temperature.

(ii) The glass transition line. The glass transition line, the blue line in Fig. 3, is the locus of the RSB continuous phase transition. As the disorder strength increases, the line moves towards higher temperatures. We find that in the crystalline state the glass line is nearly vertical (as long as we are not in the close vicinity of $T_{c}$ ) and is qualitatively the same as the single vortex depinning line depending little on the crystalline order. This is consistent with the observations of [44] where it was noticed (in the context of layered materials and columnar defects) that lateral modulation makes a very small difference to the glass line although it is obviously very important for the location of the ODO line. Consequently, four distinct phases can be identified: pinned solid (= Bragg glass), pinned liquid (= vortex glass, = amorphous solid), weakly pinned solid with marginal glassy dynamics, and unpinned liquid (or simply liquid). We compare the glass line and the ODO lines of Figs. 6 and 7 with the experimental phase diagram of a two-dimensional organic superconductor and YBCO. Shown in the inset of Fig. 6 is the magnetization jump at melting, calculated by methods of [16] and compared with the experiment of [66]. Note that the melting segment of the theoretical ODO line is negative as well as the corresponding result for YBCO presented in [16], which agrees very well with experiments of [15], while experimental curvature in the organic superconductor seems negative. The existence of the weakly pinned crystalline phase in BSCCO was firmly established thermodynamically recently [67]. The proposed phase diagram is also consistent with recent findings in both YBCO [68] and LaSCO [21].

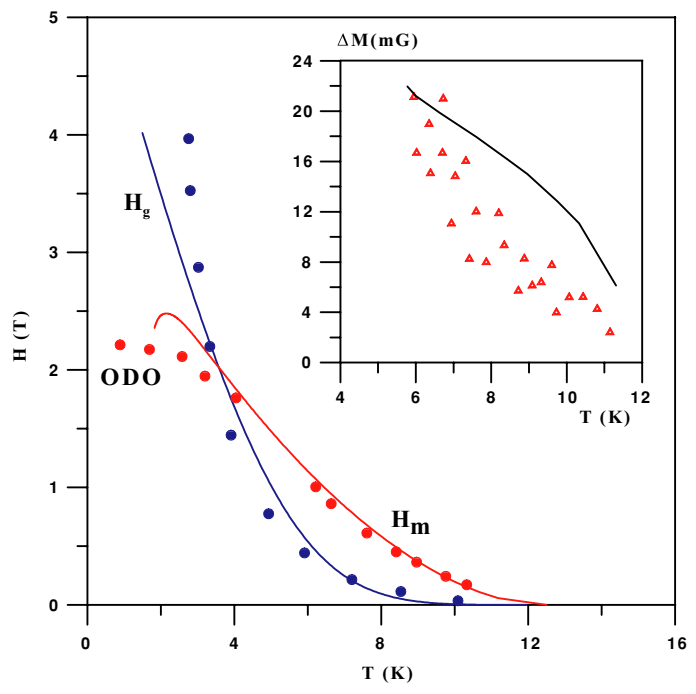

Fig. 6. Phase diagram of the organic superconductor Kappa$(B E D T-T T F)_{2} \mathrm{Cu} \cdot \mathrm{N}(\mathrm{CN})_{2} \mathrm{Br}$. Comparison of the theoretical melting line (red) and the glass line (blue) with the experimental data from [66], denoted by red and blue points, respectively. Shown in the inset is the calculated magnetization jump at melting and the corresponding experimental data. The fitting parameters are $H_{c 2}=5.5 \mathrm{~T}, T_{c}=12.5 \mathrm{~K}, \kappa=20, r=0.02, G i=0.0000015$.

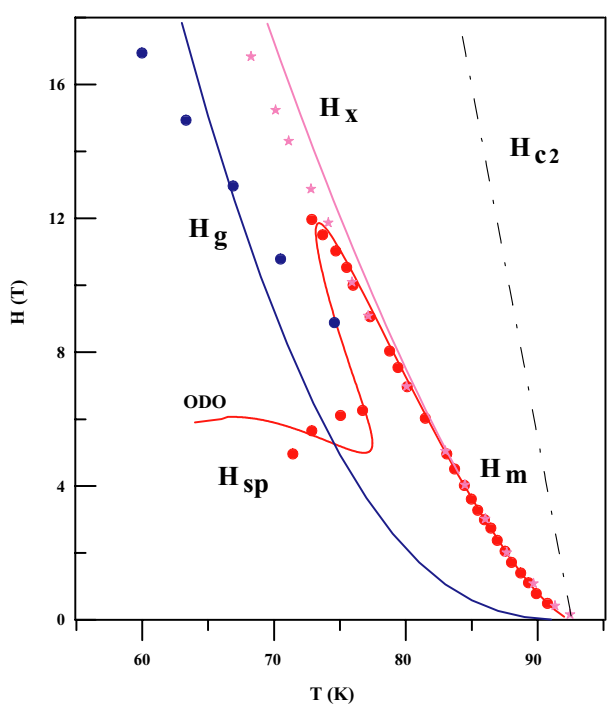

Fig. 7. Phase diagram of optimally doped YBCO. The theoretical lines, the glass line (blue), the order-disorder line (red), and the crossover between two liquid states, $H_{x}$, the pink line, are plotted together with the experimental points. The red points are the order-disorder transition points from the melting segment and from the "second magnetization peak" segment of [6], blue points are the glass transition points of [6], the pink points are the $H_{x}$ line, crossover points from [6]. The fitting parameters are $H_{c 2}=190 \mathrm{~T}$, $T_{c}=92.6, \gamma=8.3, \kappa=50, r=0.12, G i=2.010^{-4}$. 
(iii) Crossover to marginal glass. It has been recently observed $[6,20]$ that inside the liquid phase the two qualitatively different dynamical regimes take place (sometimes referred to as liquid I and liquid II). This feature receives a natural explanation within the developed description. The line separating two regimes coincides with the melting line of the pristine material, i.e., clean sample without disorder. At the same time, the analogous effect was observed in the system containing columnar defects: in this case, the transition line falls into a solid vortex system domain $[69,70]$. We now understand that in order for the glassy dynamics to set in, the two conditions are to be satisfied: (i) the system has to be in a solid phase and (ii) replica symmetry should be broken. The violation of either of this condition drives the system into the domain of the marginal dynamics. Point disorder shifts the irreversibility line to lower temperatures from the melting line. However, the effects of disorder develop at the large scale of the Larkin correlation length, while on the smaller scales the liquid preserves the crystalline structure. However, since the RSB appears only at the irreversibility line, the strip between the two lines exhibit the marginal glassy dynamics. The role of point disorder in this case is to expose the onset of the local crystalline order in the liquid. In the system with columnar defects, the loss of the symmetry breaking property occurs within the solid phase. Thus, the irreversibility line cuts the solid part of the phase diagram into two domains: (i) the lowtemperature and low-field domain with glassy dynamics due to algebraically divergent barriers and (ii) the high-temperature domain of marginal dynamics with the logarithmically divergent barriers.

\subsection{Comparison with Experiment}

These lines are compared to the glass transition lines derived from transport experimental data for the two-dimensional organic superconductor and three-dimensional high $T_{c}$ superconductor $\mathrm{YBCO}$ data in Figs. 6 and 7. At this line, the magnetization $M$ has a cusp, and its slope $d M / d T$ experiences a jump. Similar behavior was recently observed also in BSCCO [67].
The crossover line separating the two homogeneous states is the continuation of the pristine melting line and is marked by a dash-dotted (pink) " $H_{x}$ line" in Figs. 4 and 6. In Fig. 6, it is compared to the experiment by [6].

\section{SUMMARY}

To summarize, we considered the effects of both thermal fluctuations and disorder in the framework of the GL approach using the replica formalism. Flux line lattice in type II superconductors undergoes a transition into three "disordered" phases: vortex liquid (not pinned), homogeneous vortex glass (the pinned liquid or the vortex glass), and the Bragg glass (pinned solid) due to both thermal fluctuations and random quenched disorder. We show that the disordered GL (valid not very far from $H_{c 2}$ ) in which only the coefficient of a term quadratic in order parameter $\psi$ is random, first considered by Dorsey, Fisher and Huang, leads to a state with nonzero EA order parameter, but this state is still replica symmetric. Namely there is no ergodicity breaking and no multiple time scales in dynamics are expected. However, when the coefficient of the quartic term in $\psi$ in the GL free energy also has a random component, RSB effects appear (with ergodicity breaking). The location of the glass transition line in three-dimensional materials is determined and compared to experiments. The line is clearly different from both the melting line and the second peak line describing the translational and rotational symmetry breaking at high temperature and low temperature, respectively. The phase diagram is therefore separated by two lines into four phases mentioned above. In principle, we could obtain the RSB solution near the phase transition line by expanding the equations around the phase transition line as in the spin glass theory, see, for example [46], and we found that the RSB is continuous. Thus, RSB states involve multiple time scales in relaxation phenomena.

It is observed that the glass (irreversibility) line crosses the "order-disorder" line not very far from its Kauzmann point. The Kauzmann point is a point in which the magnetization and the entropy difference between solid and liquid phases changes sign. In this region, the positive slope disorder dominated second peak segment joins the thermal fluctuations dominated negative slope melting segment. This is the region in which effects of disorder and 
of the thermal fluctuation are roughly of the same strength. We thus we expect that the two GT lines (on both liquid side and solid side) have to cross right at the Kauzmann point. It would be interesting to test this idea using some simple solvable toy model.

The RSB solution we have presented can be used to calculate the detailed properties inside the glass state. This, however, would require generalization of the theory to include dynamics, since most of irreversible phenomena are time dependent. In particular, it would be interesting to estimate the time scales associated with quenched disorder. This is left for a future work. Also we have considered only the three dimensional GL model here. It can be applied to superconductors with rather small anisotropy. It would be interesting to generalize the calculation to the Lawrence-Doniach model and to the two dimensional case describing thermal fluctuations and disorder in more anisotropic layered superconductors and thin films.

\section{ACKNOWLEDGMENTS}

We are grateful to V. Yang, E.H. Brandt, E. Zeldov, B. Shapiro, G. Bel and Y. Yeshurun for numerous discussions, T. Nishizaki for providing details of their experiments. The work was supported by NSC of Taiwan grant The work of BR was supported by NSC of Taiwan grant NSC \#93-2112-M009-023 and the work of DL was supported by National Nature Science Foundation (\#90403002).

\section{REFERENCES}

1. D. R. Nelson, Phys. Rev. Lett. 60, 1973 (1988).

2. J. Blatter, M. V. Feigelman, V. B. Geshkenbein, A. I. Larkin, and V. M. Vinokur, Vortices in high temperature superconductors, Rev. Modern Phys. 66, 1125 (1994)

3. P. Gammel, D. A. Huse, and D. J. Bishop, Spin Glasses and Random Fields, A. P. Young, ed. (World Scientific. Singapore, 1998)

4. J. Kierfeld and V. Vinokur, Phys. Rev. B 61, R14928-R14931 (2000)

5. G. P. Mikitik and E. H. Brandt, Phys. Rev. B 64, 184514 (2001).

6. F. Bouquet, C. Marcenat, E. Steep, R. Calemezuk, W. K. Kwok, U. Welp, G. W. Crabtree, R. A. Fisher, N. E. Phillips, and A. Schilling, Nature 411, 448 (2001).

7. T. Giamarchi and P. Le Doussal, Phys. Rev. Lett. 75, 3372 (1994); Phys. Rev. B 52, 1242 (1995).

8. Ertas and D. R. Nelson, Physica C 272, 79 (1996).

9. V. Vinokur, B. Khaykovich, E. Zeldov, M. Konczykowski, R. A. Doyle, and P. H. Kes, Physica C 295(3-4), 209-217 (1998)
10. E. Zeldov, D. Majer, M. Konczykowski, V. B. Geshkenbein, V. M. Vinokur, and H. Shtrikman, Nature 375, 373 (1995); R. Liang, D. A. Bonn, and W. N. Hardy, Phys. Rev. Lett. 76, 835 (1996).

11. A. Schilling, R. A. Fisher, N. E. Phillips, U. Welp, D. Dasgupta, W. K. Kwok, and G. W. Crabtree, Nature 382, 791 (1996); M. Roulin, A. Junod, and E. Walker, Science 273, 1210 (1996).

12. B. Khaykovich, E. Zeldov, D. Majer, T. W. Li, P. H. Kes, and M. Konczykowski, Phys. Rev. Lett. 76, 2555 (1996).

13. Y. Radzyner, A. Shaulov, and Y. Yeshurun, Phys. Rev. B 65, 100513 (R) (2002); Y. Radzyner, A. Shaulov, Y. Yeshurun, I. Felner, K. Kishio, and J. Shimoyama, Phys. Rev. B 65, 100503 (R) (2002)

14. N. Avraham, B. Khaykovich, Y. Myasoedov, M. Rappaport, H. Shtrikman, D. E. Feldman, T. Tamegai, P. H. Kes, M. Li, M. Konczykowski, K. van der Beek, and E. Zeldov, Nature 411, 451 (2001).

15. N. Kobayashi, T. Nishizaki, K. Shibata, T. Sato, M. Maki, and T. Sasaki, Physica C 362, 121 (2001); K. Shibata, T. Nishizaki, T. Sasaki, and N. Kobayashi, Phys. Rev. B 66, 214518 (2002); M. Willemin et al., Phys. Rev. Lett. 81, 4236 (1998).

16. D. Li and B. Rosenstein, Phys. Rev. Lett. 90, 167004 (2003); Phys. Rev. B 70, 144521 (2004).

17. D. Pal, S. Ramakrishnan, A. K. Grover, D. Dasgupta, and Bimal K. Sarma, Phys. Rev. B 63, 132505 (2001); D. Pal, S. Ramakrishnan, and A. K. Grover, Phys. Rev. B 65, 096502 (2002).

18. K. Deligiannis, M. Charalambous, J. Chaussy, R. Liang, D. Bonn, and W. N. Hardy, Physica C 341, 1329 (2000).

19. B. J. Taylor, S. Li, M. B. Maple, and M. P. Maley, Phys. Rev. $B$ 68, 054523 (2003).

20. D. T. Fuchs, E. Zeldov, T. Tamegai, S. Ooi, M. Rappaport, and H. Shtrikman, Phys. Rev. Lett. 80, 4971 (1998); E. Zeldov, private communication.

21. U. Divakar, A. J. Drew, S. L. Lee, R. Gilardi, J. Mesot, F. Y. Ogrin, D. Charalambous, E. M. Forgan, G. I. Menon, N. Momono, M. Oda, C. D. Dewhurst, and C. Baines, Phys. Rev. Lett. 92, 237004 (2004); T. Sasagawa, Y. Togawa, J. Shimoyama, A. Kapitulnik, K. Kitazawa, and K. Kishio, Phys. Rev. B 61, 1610 (2000).

22. V. Dotseko, An Introduction to the Theory of Spin Glasses and Neural Networks (Cambridge University Press, Cambridge, 2001).

23. L. B. loffe and V. M. Vinokur, J. Phys. C 20, 6149 (1987).

24. M. P. A. Fisher, Phys. Rev. Lett. 62, 1415 (1989); D. S. Fisher, M. P. A. Fisher, and D. A. Huse, Phys. Rev. B 43, 130 (1991).

25. M. V. Feigel'man, V. B. Geshkenbein, A. 1. Larkin, and V. M. Vinokur, Phys. Rev. Lett. 63, 2303 (1989).

26. T. Nattermann, Phys. Rev. Lett. 64, 2454 (1990).

27. A. T. Dorsey, M. Huang, and M. P. A. Fisher, Phys. Rev. B 45, 523 (1992).

28. O. Narayan and M. Fisher, Phys. Rev. B 48, 7030 (1993).

29. M. Dong et al., Phys. Rev. Lett. 70, 662 (1993).

30. L. V. Micheev, B. Drossel, and M. Kardar, Phys. Rev. Lett. 75, 1170 (1995).

31. V. M. Vinokur, M. C. Marchetti, and L.-W. Chen, Phys. Rev. Lett. 77, 1845 (1996).

32. P. Chauve, T. Giamarchi, and P. Le Doussal, Europhys. Lett. 44, 110 (1998).

33. A. van Otterlo, R. T. Scalettar, and G. T. Zimanyi, Phys. Rev. Lett. 81, 1497 (1998).

34. T. Natterman and S. Scheindl, Adv. Phys. 49, 607 (2000).

35. Y. Nonomura and X. Hu, Phys. Rev. Lett. 28, 5140 (2001).

36. P. Olsson and S. Teitel, Phys. Rev. Lett. 87, 1370001 (2001).

37. T. Giamarchi and S. Bhattacharya, in High Magnetic Fields: Applications in Condensed Matter Physics and Spectroscopy C. Berthier et al., eds. (Springer-Verlag, Berlin, 2002) p. 314.

38. H. K. Bokil and A. P. Young, Phys. Rev. Lett. 74, 3021 (1995). 
39. C. Reichhardt, A. van Otterlo, and G. T. Zimanyi, Phys. Rev. Lett. 84, 1994 (2000).

40. S. E. Korshunov, Phys. Rev. B 48, 3969 (1993).

41. E. H. Brandt, Rep. Prog. Phys. 58, 1465 (1995).

42. D. J. Thouless, Phys. Rev. Lett. 34, 946 (1975); E. Brezin, D. R. Nelson, and A. Thiaville, Phys. Rev. B 31, 7124 (1985).

43. H. Sompolinsky and A. Zippelius, Phys. Rev. B 25, 6860 (1982).

44. Z. Tesanovie and I. F. Herbut, Phys. Rev. B 50, 10389 (1994).

45. A. V. Lopatin, Europhys. Lett. 51, 635 (2000).

46. G. Parisi, J. Phy. A 13, 1101 (1980).

47. D. Li, B. Rosenstein, Phys. Rev. Lett. 86, 3618 (2001); Phys. Rev. B 65, R220504, 024514 (2002).

48. A. Schilling, U. Welp, W. K. Kwok, and G. W. Crabtree, Phys. Rev. B 65, 054505 (2002).

49. G. I. Menon and C. Dasgupta, Phys. Rev. Lett. 73, 1023 (1994); G. I. Menon, C. Dasgupta, and T. V. Ramakrishnan, Phys. Rev. B 60, 7607 (1999).

50. G. J. Ruggeri and D. J. Thouless, J. Phys. F 6, 2063 (1976); Z. Tesanovic and A. V. Andreev, Phys. Rev. B 49, 4064 (1994); R. Săik and D. Stroud, Phys. Rev. Lett. 75, 2582 (1995).

51. T. Giamarchi, and P. Le Doussal, Phys. Rev. B 53, 15206 (1996); Phys. Rev. B 55, 6577 (1997).

52. M. Mezard and G. Parisi, J. Phys. (Fr.) 11, 809 (1991).

53. Z. L. Xiao, E. Y. Andrei, and M. J. Higgins, Phys. Rev. Lett. 83, 1664 (1999); Y. Paltiel, E. Zeldov, Y. N. Myasoedov, H. Shtrikman, S. Bhattacharya, M. J. Higgins, Z. L. Xiao, E. Y. Andrei, P. L. Gammel, and D. J. Bishop, Nature 403, 398 (2000); Y. Paltiel, E. Zeldov, Y. Myasoedov, M. L. Rappaport, G. Jung, S. Bhattacharya, M. J. Higgins, Z. L. Xiao, E. Y. Andrei, P. L. Gammel, and D. J. Bishop, Phys. Rev. Lett. 85, 3712 (2000); X. S. Ling, S. R. Park, B. A. McClain, S. M. Choi, D. C. Dender, and J. W. Lynn, Phys. Rev. Lett. 86, 712 (2001); Z. L. Xiao, O. Dogru, E. Y. Andrei, P. Shuk, and M. Greenblatt, Phys. Rev. Lett. 92, 227004 (2004).
54. J. R. L. de Alameida and D. J. Thouless, J. Phys. All, 983 (1978).

55. K. Binder and A. P. Young, Rev. Modern Phys. 58, 801 (1986).

56. J. P. Bouchaud and M. Mezard, J. Phys. A: Math. Gen. 307997 (1997).

57. B. Drossel, J. Stat. Phys. 82, 431 (1996).

58. R. Rammal, J. Physique 46, 1837 (1985).

59. M. Z. Bazant, cond-mat/9905191.

60. D. S. Fisher, P. Le Doussal, and C. Monthus, Phys. Rev. Lett. 80, 3539 (1998); P. Le Doussal, C. Monthus, and D. S. Fisher, Phys. Rev. E 59, 4795 (1999).

61. E. J. Gumbel, Statistical Theory of Extreme Values and Some Practical Applications (National Burcau of Standards Applied Mathematics Series, vol. 33, 1954); Statistics of Extrmes (Columbia University Press, New York, 1958).

62. R. A. Fisher and L. H. C. Tippett, Proc. Camb. Philos. Soc. 24, 180 (1928).

63. B. V. Gnedenko and A. N. Kolmogorov, Limit distributions for Sums of Independent Random Variables (Addison Wesley, Reading, MA, 1954).

64. J. Galambos, The Asymptotic Theory of Extreme Order Statistics (Wiley, New York, 1987).

65. H. J. Herrmann and H. E. Stanley, Phys. Rev. Lett. 53, 1121 (1984); H. A. Makse and L. A. Nunes Amaral, Europhys. Lett. 31, 379 (1995).

66. T. Shibauchi, M. Sato, S. Ooi, and T. Tamegai, Phys. Rev. B 57, 5622 (1998).

67. H. Biedenkopf, et al., Phys. Rev. Lett. 95, 257004 (2005).

68. D. Giler, A. Shaulov, Y. Yeshurun, and J. Giapintzakis, Phys. Rev. B 60, 106 (1999); B. J. Taylor, S. Li, M. B. Maple, and M. P. Maley, Phys. Rev. B 68, 054523 (2003).

69. A. V. Lopatin and V. M. Vinokur, Phys. Rev. Lett. 92, 067008 (2004).

70. S. Colson, et al. Phys. Rev. B 69, 180510 (2004). 